\title{
The effect of flow oscillations on cavity drag
}

\author{
By M. GHARIB $\uparrow$ AND A. ROSHKO \\ Graduate Aeronautical Laboratories, California Institute of Technology, \\ Pasadena, CA 91 125, USA
}

(Received 11 March 1986 and in revised form 10 September 1986)

An experimental investigation of flow over an axisymmetric cavity shows that self-sustained, periodic oscillations of the cavity shear layer are associated with low cavity drag. In this low-drag mode the flow regulates itself to fix the mean-shear-layer stagnation point at the downstream corner. Above a critical value of the cavity width-to-depth ratio there is an abrupt and large increase of drag due to the onset of the 'wake mode' of instability. It is also shown by measurement of the momentum balance how the drag of the cavity is related to the state of the shear layer, as defined by the mean momentum transport $\rho \bar{u} \bar{v}$ and the Reynolds stress $\rho \overline{u^{\prime} v^{\prime}}$, and how these are related to the amplifying oscillations in the shear layer. The cavity shear layer is found to be different, in several respects, from a free shear layer.

\section{Introduction}

The flow over a 'cavity' or cutout in a flat surface has attracted the attention of many investigators, largely no doubt because of its geometrical simplicity but also because of its relevance to many practical flow problems. As in many other flows (e.g. the flow past a circular cylinder), geometrical simplicity does not necessarily ensure flow simplicity and, indeed, the cavity flows have provided an assortment of interesting theoretical questions and experimental observations. The latter include the phenomenon of 'cavity-oscillation'. Other, possibly related, aspects of the flow include the mechanics of the separated shear layer; the internal recirculating flow, sometimes called the 'cavity-vortex'; the changes of flow regime with changing geometry; and, not least, the 'drag' of the cavity. These various aspects have been addressed by many investigators.

It was our objective in the experiments described here to see how the drag is related to the properties of the separated shear layer and how these are influenced by the well defined, laminar, periodic oscillations that are known to exist at particular combinations of geometry and Reynolds number.

A simple example of cavity flow is given along with its physically significant parameters in figure 1. It is known (Karamcheti 1956; Sarohia 1975) that for initiation of self-sustained oscillation of the cavity shear layer a minimum cavity width $\left(b_{\min }\right)$ is required. Determination of $b_{\min }$ will depend on the flow parameters $U_{\mathrm{e}}, \theta_{0}$ and $\nu$ where $U_{\mathrm{e}}$ is the mean velocity outside the cavity and $\theta_{0}$ is the momentum thickness of the boundary layer at the upstream corner and $\nu$ is the kinematic viscosity of the fluid.

Sarohia (1975), in his study of the frequency selection process, following earlier work of Woolley \& Karamcheti (1973), concluded that the frequency of cavity-flow

$\uparrow$ Present address: Department of Applied Mechanics and Engineering Sciences, University of California, San Diego, La Jolla, California 92093, USA. 


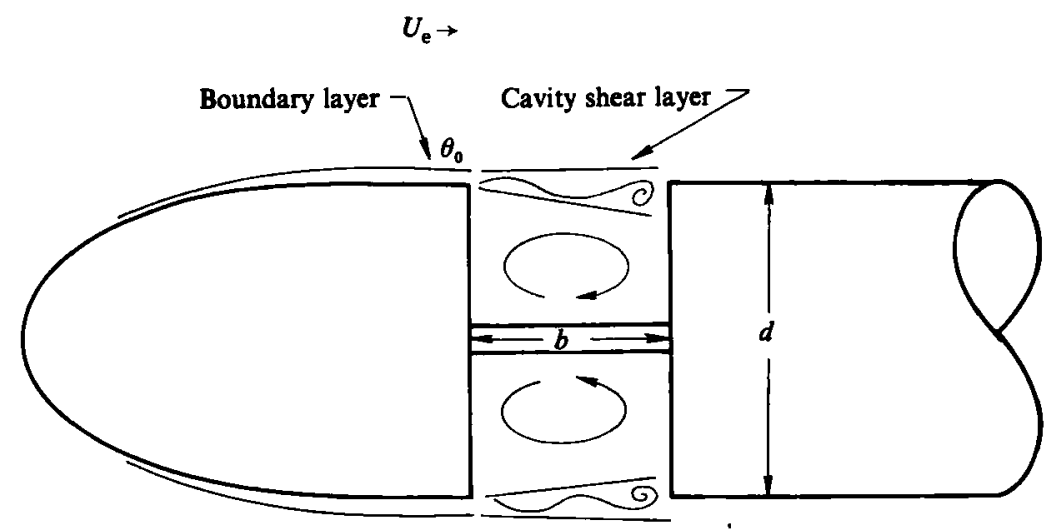

Figure 1. Schematics of the cavity model and the flow.

oscillation which is selected is such that (i) certain phase criteria are satisfied; and (ii) maximum integrated amplification along the cavity shear layer occurs.

The final amplitude growth at the selected frequency results in the roll-up of the shear layer and appearance of large vortical structures similar to those in the free shear layers observed by Freymuth (1966). Rockwell \& Naudascher (1978) suggest that impingement of the large vortical structures on the downstream corner influences the sensitive region of the shear layer close to the upstream corner through the Biot-Savart induction law. The reception and conversion of this upstream influence into velocity fluctuations which is followed, with further amplification and growth, by development of vortex structures, completes the cycle of events necessary for the self-sustainment of the oscillations. The frequency of oscillation decreases as the cavity width $b$ increases (Sarohia 1975), then jumps to a higher frequency and starts another similar reduction of frequency in the new, higher mode. Eventually, after the cavity width exceeds a certain value of $b$, for given depth, the flow loses the ability to oscillate spontaneously in a cavity mode and develops fluctuations on a larger scale, related to the depth of the cavity rather than the thickness of the shear layer. It was this that motivated the present investigation into the role of instabilities in establishing the pressure field and drag of the cavities. It was felt that an understanding of the mechanism for the transfer of momentum into and out of the cavity is necessary to explain some of the phenomena that occur in this type of flow. The magnitude and distribution of the mean momentum transfer $(\overline{u v})$ and the Reynolds stress $\left(\overline{u^{\prime} v^{\prime}}\right)$ along the cavity shear layer play a central role in the determination of the drag of the system. The main objectives were to understand the effect of instability growth in the layer on these two terms and then to relate them to the drag of the cavity.

\section{Experimental details}

The experiments were performed in a newly constructed free-surface recirculating water tunnel (Gharib 1983), which is located at the Jet Propulsion Laboratory, California Institute of Technology. This tunnel is specially designed to attain a low turbulence level for the purpose of carrying out sensitive, flow-instability-type experiments. The test section is $45.7 \mathrm{~cm}$ (18 in.) wide by $45.7 \mathrm{~cm}$ (18 in.) deep. The wetted depth can be increased up to $56 \mathrm{~cm}(22 \mathrm{in}$.). The test section is enclosed by 
glass windows on sides, bottom and top to permit visual and optical study of the flow throughout its $1.83 \mathrm{~m}(6 \mathrm{ft})$ length. It is possible to achieve a maximum velocity of $1 \mathrm{~m} / \mathrm{s}$ in the test section. The free-stream wide-band turbulence level at $25 \mathrm{~cm} / \mathrm{s}$ on the centreline is near $0.05 \%$.

In this study, the cavity was an annular gap downstream of the 4 in. half-ellipsoidal nose of a 4 in. diameter axisymmetric body which was aligned parallel to the flow in the water channel. The cylindrical geometry avoids the often troublesome problem of end effects in the two-dimensional separated flows. The nose of the model was connected to the rear portion by a sting as shown schematically in figure 1 . The rear portion is a hollow cylinder 4 in. in diameter and 36 in. in length; it was connected by a strut to a traversing mechanism installed above the test section.

The model was equipped with two dye-injection ports $2.54 \mathrm{~cm} \mathrm{(1} \mathrm{in.)} \mathrm{from} \mathrm{the}$ stagnation point of the model nose. To visualize the flow either food-colouring dye or fluorescein dye was gravity fed to the injection tubes and thus discharged into the boundary layer over the cavity.

When using blue dye, a uniform background illumination was used for photographic purposes. The fluorescein, however, was illuminated and excited by a sheet of laser light less than $1 \mathrm{~mm}$ thick obtained from a 2 Watt argon-ion laser. This technique was used first by Dewey (1976) and more recently by Dimotakis, Miake-Lye \& Papantoniou (1983).

The solid inner faces of the cavity were equipped with pressure taps, $1 \mathrm{~mm}$ in diameter, 14 on the downstream face and 8 on the upstream face.

\subsection{Instrumentation}

The low velocities in the range of interest introduced the well-known difficulty of measuring low differential pressures under high head. A free-stream velocity of $25 \mathrm{~cm} / \mathrm{s}$ generates a dynamic head of only $0.38 \mathrm{~cm}(0.15 \mathrm{in}$.) of water, while a typical hydro-static head at the pressure transducer location is $63.5 \mathrm{~cm} \mathrm{(25} \mathrm{in.)} \mathrm{of} \mathrm{water.}$ Further, since differential static pressures inside the cavity are even smaller than the free-stream dynamic head, the demand on the pressure measuring system for high resolution and repeatability is very severe. A pressure transducer (Validyne type Wet-Wet DP-103-12) which was able to measure differential pressures in the range of 0 to $0.762 \mathrm{~cm}(0.3 \mathrm{in}$.) of water with a resolution of $1 \%$ of the total range was used in conjunction with a 24 port fast-switch (Scanivalve type W24-60).

For the purpose of spectral analysis and phase measurement, velocity fluctuation measurements were made with a TSI hot-film probe (1277-10A) and a TSI 1050C anemometer in conjunction with a TSI true r.m.s. meter (1076). The output of the hot-film anemometer was fed to a digital power spectrum analyser (Schulemberg SL20). A bandwidth of $0.1 \mathrm{~Hz}$ was used for locating and distinguishing frequency content of the velocity signals.

For phase-angle and cross-correlation measurements a second hot-film probe was mounted on a traversing mechanism which was able to traverse the entire width of the cavity. Throughout this experiment the first probe was used as a reference probe. It was placed above the upstream corner at $x=b$ and $y_{0.95}$, the latter being the $y$-location of $\bar{u} / U_{\mathrm{e}}=0.95$. Phase measurements were made by moving the second probe relative to the reference probe along the edge of the shear layer where $\bar{u} / U_{\mathrm{e}}=0.95\left(y=y_{0.95}\right)$. The signals from the reference and the second probe were fed to a digital probability and correlation analyser (SAICOR 43A). Throughout the phase-angle or cross-correlation measurements a sampling rate of 200 per second, limited by 400 samples and a corresponding Nyquist frequency of about 30 , was used. 


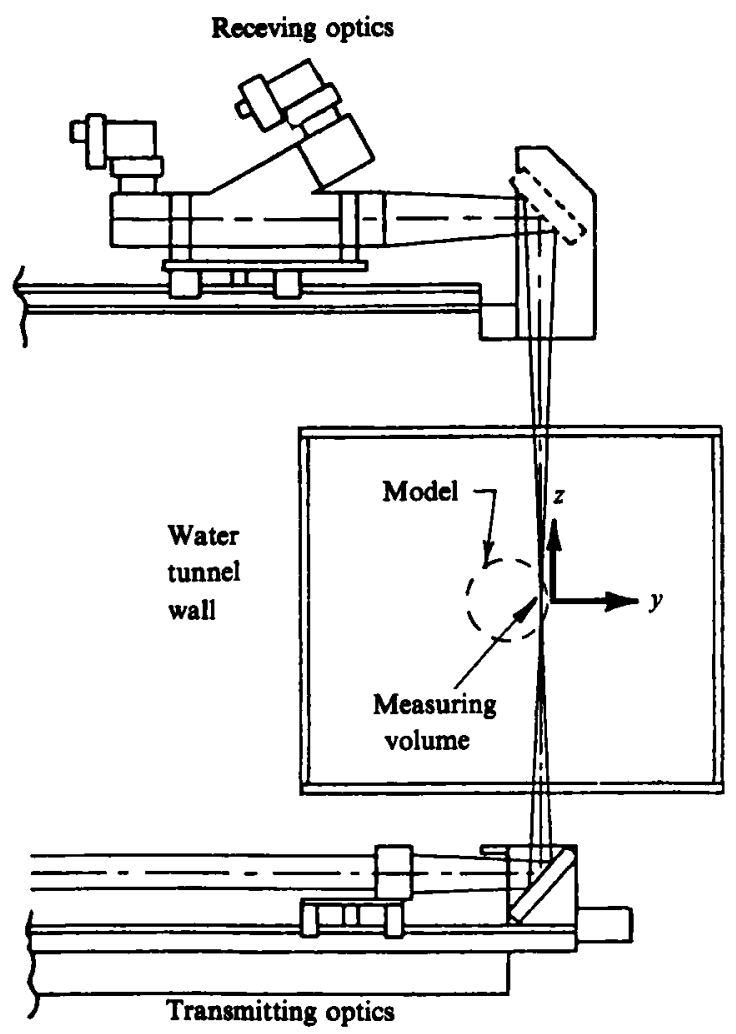

FraURE 2. Schematics of the laser-Doppler velocimeter set-up.

The velocity field of the cavity flow was measured by a two-component, two-colour, frequency-shifted laser-Doppler velocimeter (TSI 9100-7 system). A dual-beam forward-scatter configuration was used, with the transmitting and receiving optics located at the bottom and top side of the facility as shown in figure 2. The physical arrangement of the LDV optics relative to the facility and information regarding focal volume are given in figure 2. A velocity-bias technique (Bragg shifting) was used to resolve the ambiguity problem in regions of velocity reversal. Two counter processors, one for each velocity component, were operated in the single-burst mode, for which only one particle is present in the focal volume when the measurement is made. In addition, the system was operated in coincident mode, in which the output of the processors is tested for coincidence in time so that the two components of the velocity vector, as determined by the optical set-up, are measured on the same particle. A coincidence window of $50 \mu \mathrm{s}$ was used in all velocity measurements. An LDV processor was operated in conjunction with a minicomputer system (PDP-11), which was also used for processing the results. The amplitude threshold level of the processors and the gain of the receiving amplifiers were adjusted to maintain the data rate between 60 and 200 particles/s, which corresponds to a minimum Nyquist frequency of 10 . The size of the data buffer combined with the data rate gave a total sampling time between 15 and $60 \mathrm{~s}$. This sampling time was considered adequate to properly characterize the mean turbulent quantities. At every measurement point, a frequency histogram was constructed for each channel. This histogram provided 
information about the proper operation of the system, i.e. filter setting, and the electronic noise.

From the data mean values, second moments and cross-correlation coefficients of the two velocity components were evaluated and from them the mean momentum transfer $(\overline{u v})$ and Reynolds stress $\left(\overline{u^{\prime} v^{\prime}}\right)$ were obtained.

\subsection{Operational conditions and reference definitions}

Measurements were made at speeds covering the range $16-27 \mathrm{~cm} / \mathrm{s}$. The corresponding Reynolds number, $R e$, based on the model diameter, $d=10.16 \mathrm{~cm}(4 \mathrm{in}$.) and at $U_{\mathrm{e}}=23 \mathrm{~cm} / \mathrm{s}$, at which most of the tests were performed, is $2.4 \times 10^{4}$.

The origin of the reference coordinate system is located at the upstream corner of the cavity. The $x$-axis is in the direction of flow and the $y$-axis is in the radial direction (figure 2). The components of velocity vector in the direction of the $x$ - and $y$-axes are denoted by $u$ and $v$.

The cavity width, $b$, is normalized with respect to $\theta_{0}$, the momentum thickness of the upstream boundary layer at the point of separation. The momentum thickness for the shear layer is defined as

$$
\theta=\int_{+\infty}^{-\infty} \frac{\bar{u}}{U_{\mathrm{e}}}\left(1-\frac{\bar{u}}{U_{\mathrm{e}}}\right) \mathrm{d} y
$$

where the limits correspond to $\mathrm{d} \bar{u} / \mathrm{d} y=0$ and $U_{\mathrm{e}}$ is the velocity on the outside edge of the boundary layer at the point of separation. Using $U_{\mathrm{e}}$ instead of a reference velocity upstream of the model eliminates the need for blockage correction.

At $U_{\mathrm{e}}=23 \mathrm{~cm} / \mathrm{s}$ the boundary layer upstream of the cavity was laminar and had a shape factor $H=\delta_{0} / \theta_{0}=2.5$ where $\delta_{0}=1.00 \mathrm{~mm}$ (displacement thickness), $\theta_{0}=0.41 \mathrm{~mm}$ and $R e_{\theta_{0}}=95$. For the shear layer, the transverse distance $y$ is non-dimensionalized as $\left(y-y_{0.5}\right) / \theta$, where $y_{0.5}$ is the location at which the mean velocity, $\bar{u}$, has a value of $0.5 U_{\mathrm{e}}$. The radius of the axisymmetric cavity, which is $5.08 \mathrm{~cm}(2 \mathrm{in}$.$) , is used to normalize the radial distance of pressure taps (r)$ on the inner surfaces of the cavity.

\section{Characteristics of the cavity shear layer}

The purpose of the measurements presented in this section was to characterize the cavity free shear layer as completely as possible and to provide solid reference conditions for the cavity-drag measurements to be presented in $\$ 4$.

\subsection{Self-sustained oscillations}

For small cavity gaps, i.e. $b / \theta_{0}<b / \theta_{0_{\min }}$, the shear layer smoothly bridges over the cavity with no distinct oscillation in it. Intermittent bursts of periodic oscillation may occur as the cavity width approaches the required minimum value for the onset of the oscillation. A self-sustained oscillation appears in the cavity shear layer when $b / \theta_{0}$ reaches $b / \theta_{0 \min }$. The oscillation is always associated with the presence of a distinct fundamental frequency in the power spectrum of the velocity fluctuations (figure 3). The fundamental frequency was identified as the one that has the highest peak in the power spectrum. The variation of fundamental frequency with $b / \theta_{0}$ at a fixed edge velocity $\left(U_{\mathrm{e}}=23 \mathrm{~cm} / \mathrm{s}\right)$ is shown in figure 4 . Oscillation starts at $b / \theta_{0} \approx 81$, which was the minimum cavity width, $b / \theta_{0_{\min }}$ for these experimental conditions. For reasons explained below, we identify this as a mode II oscillation. 


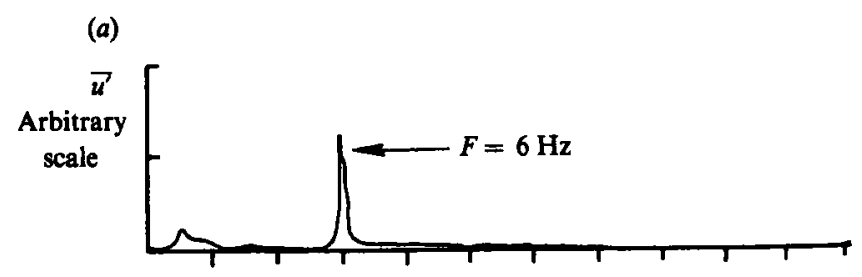

(b)

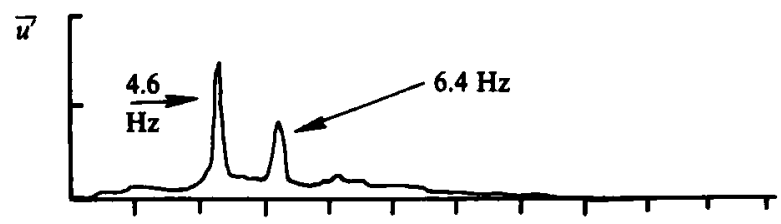

(c)

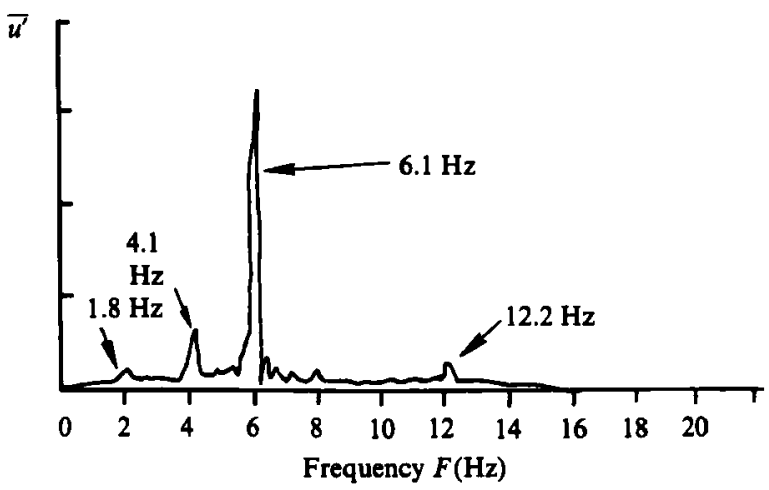

FiaURe 3. Velocity fluctuation spectra for increasing cavity width from mode II to mode III, obtained at $Y_{0.95}$. (a) $b / \theta_{0}=84$ mode II; $(b) b / \theta_{0}=122$ transition; $(c) b / \theta_{0}=134$ mode III.

The fundamental frequency of oscillation decreases as $b / \theta_{0}$ increases. This course of frequency variation is interrupted by a sudden jump of frequency from 4.6 to 6. $2 \mathrm{~Hz}$. The latter is in mode III. As $b / \theta_{0}$ further increases, the frequency decreases from its new value. A region of hysteresis, approximately as shown in figure 4 , is associated with the transition region. The oscillations in the hysteresis region often show intermittent switching between the two modes.

Figure 5 depicts the variation of overall phase difference $\phi$ between the two corners of the cavity. This was measured between two probes located near the outside edge of the shear layer (locus of $\bar{u} / \bar{U}_{\mathbf{e}}=0.95$ ) following Ziada \& Rockwell (1981). By tracking one of the probes along the shear layer, keeping the other one fixed at $x=b$, it was possible to determine the wavelength $\lambda$ and thus the phase velocity $U_{\mathrm{c}}$. These quantities satisfy the relation

$$
\frac{\phi}{2 \pi}=\frac{b}{\lambda}=\frac{f b}{U_{\mathrm{c}}}=N,
$$

where $N$ is the number of wavelengths of fundamental frequency contained by the cavity width in the $N$ th mode of oscillation, $\lambda$ is the wavelength of the fundamental frequency and $U_{\mathrm{c}}$ is the phase velocity of the fundamental frequency. A significant 


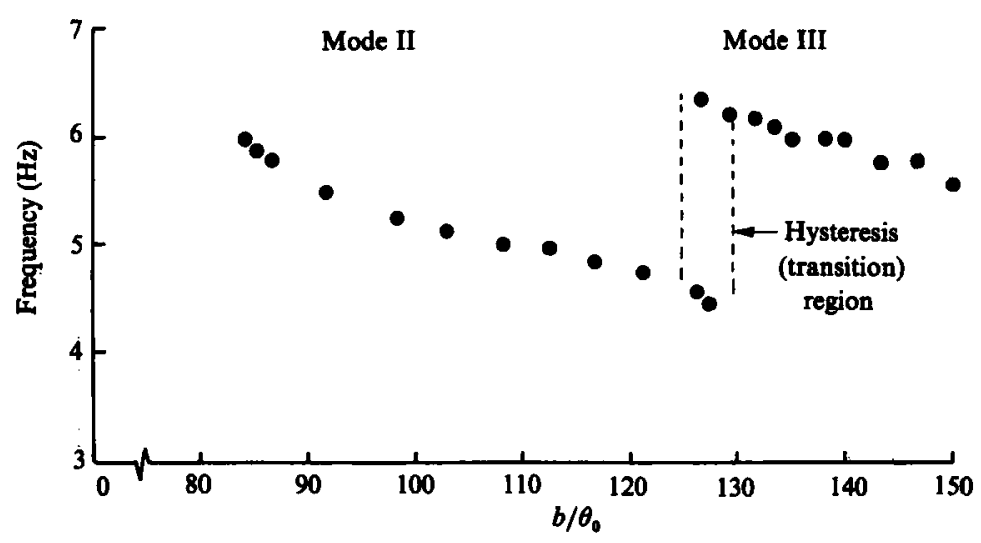

Figure 4. Frequency variation of cavity shear layer with varying cavity width, constant free-stream velocity.

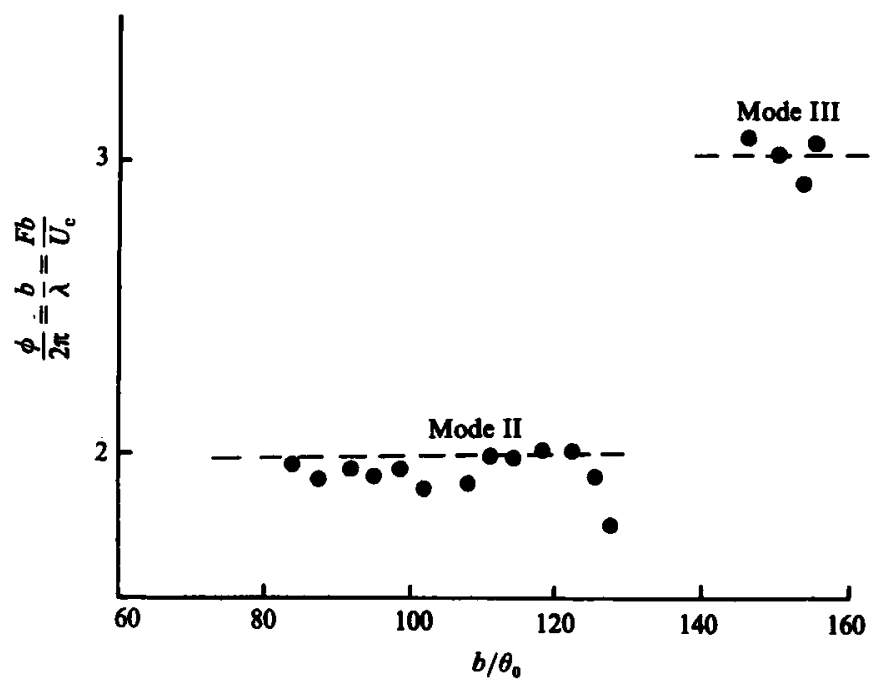

FIgURE 5. Overall phase difference between two corners of the cavity for mode II and III of the cavity-flow oscillations.

deviation from $\phi=2 \pi N$ occurs near the jump between modes. The relation (3.1) is only valid for the limit of the incompressible flow.

The flow visualization of the cavity shear layer (figure 6) confirms that in mode II of the oscillation there are two wavelengths (or vortical structures) contained in the cavity, while in mode III there are three waves (or vortical structures).

In the present study, the cavity always started the oscillation in mode II and finished in mode III, omitting the first mode $\phi / 2 \pi=b / \lambda=f b / U_{c}=1$. Sarohia (1975) observed similar omissions of the first mode for some free-stream velocities. The last mode of oscillation (mode III) shows strong intermittency as the cavity width is increased; its end at $b / \theta_{0} \approx 155$ is marked by the disappearance of the fundamental frequency. Flow visualization studies (figure 7 ) indicate that the axisymmetric cavity shear layer of the self-sustained oscillation regime becomes highly asymmetric and no longer reattaches onto the downstream corner. The cavity 
(a)

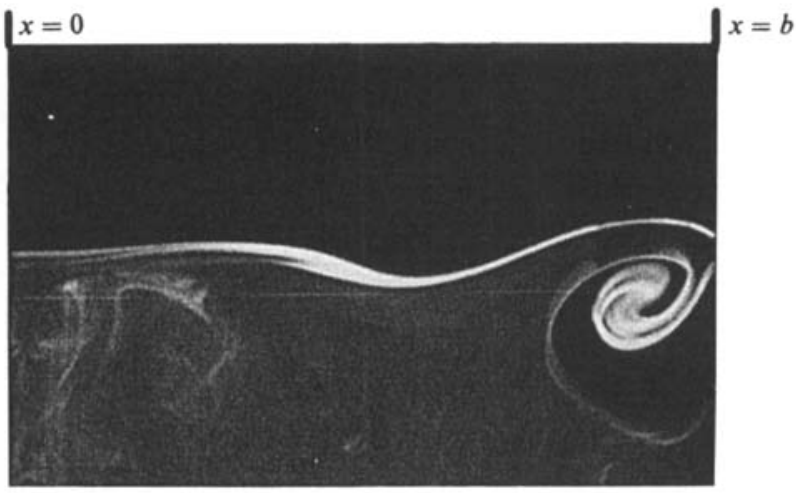

(b)

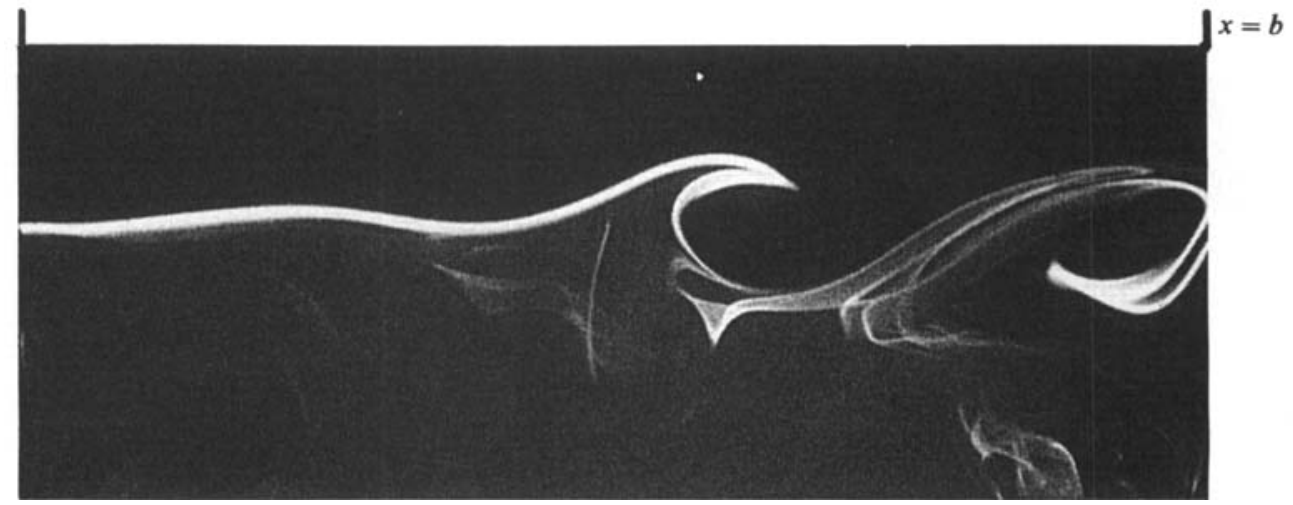

Figure 6. Flow visualization of the cavity shear layer; flow is frozen at $\frac{1}{1000}$ s. (a) Mode II $b / \lambda=F b / U_{\mathrm{c}}=2$. (b) Mode III $b / \lambda=F b / U_{\mathrm{c}}=3$.

flow becomes unstable on a large scale, comparable to the diameter of the model. A flow visualization of this case (figure 7) shows that the flow reattaches below the downstream corner. The flow field in this regime begins to show some resemblance to a three-dimensional bluff-body wake (Werle 1974), but of course with the rear body strongly interacting with and influencing the wake of the front body. A similar behaviour for the wakes of disks positioned in front of a circular cylinder in tandem, with sufficiently high values of $b / d$, was described by Koenig \& Roshko (1985). In the present study, this stage of the cavity flow field will be called the 'wake mode'.

\subsection{Mean velocity distributions in the cavity shear layer}

For three values of cavity width $b / \theta_{0}=85, b / \theta_{0}=103$ and $b / \theta_{0}=130$, mean velocity profiles were obtained at a number of streamwise stations. With edge velocity $U_{\mathrm{e}}$ fixed at $23 \mathrm{~cm} / \mathrm{s}$, the cavity width $b / \theta_{0}=85$ marks the beginning of mode II $\left(F b / U_{\mathrm{c}}=2\right)$; for $b / \theta_{0}=130\left(F b / U_{\mathrm{c}}=3\right)$ the cavity shear layer was oscillating in mode III. Figure 8 presents mean-velocity profiles, $\bar{u} / U_{\mathrm{e}}$, at selected downstream stations for these three cases. As shown in figure 8 , in the early stages of the shear-layer growth, the velocity profile changes from a boundary-layer profile to a shear-layer profile. For four values of cavity width, $b / \theta_{0}=85$ (mode II), $b / \theta_{0}=103$ (mode II), $b / \theta_{0}=119\left(\operatorname{mode}\right.$ II) and $b / \theta_{0}=135$ (mode III), the growth 
(a)

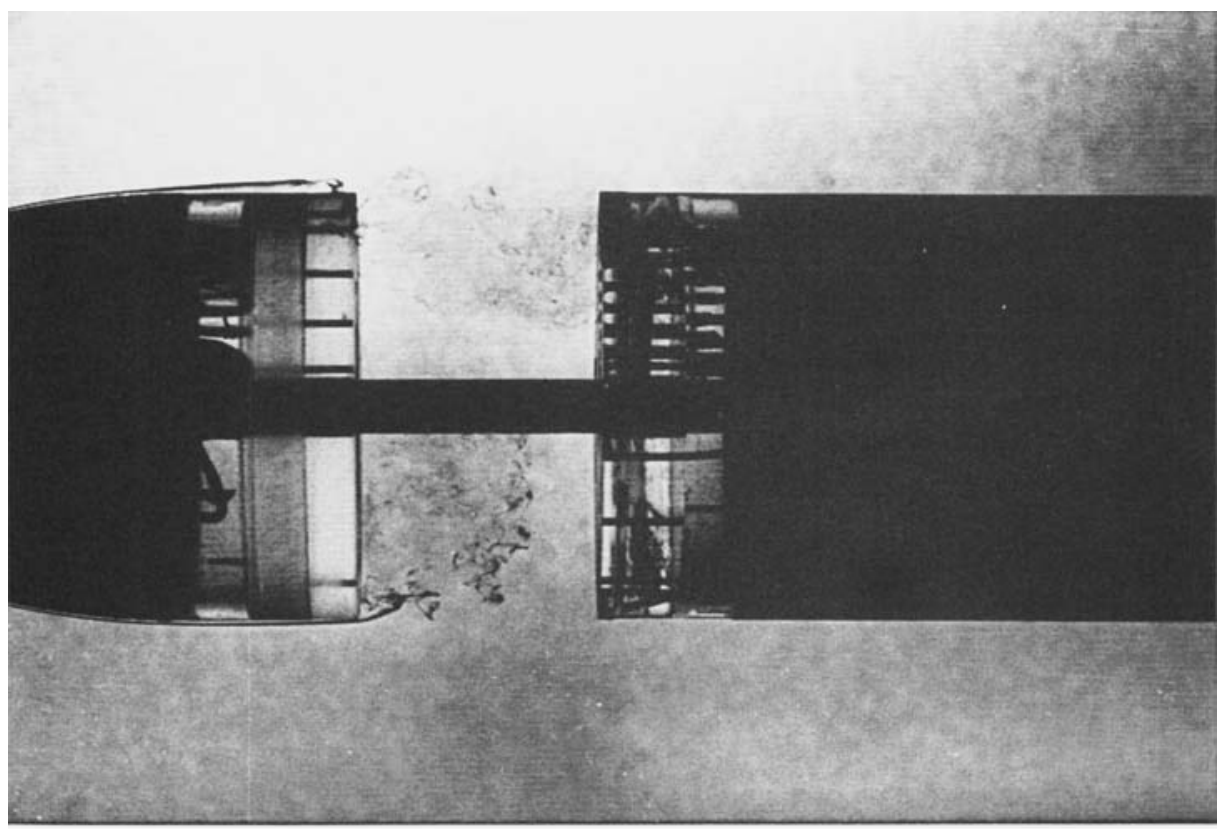

(b)

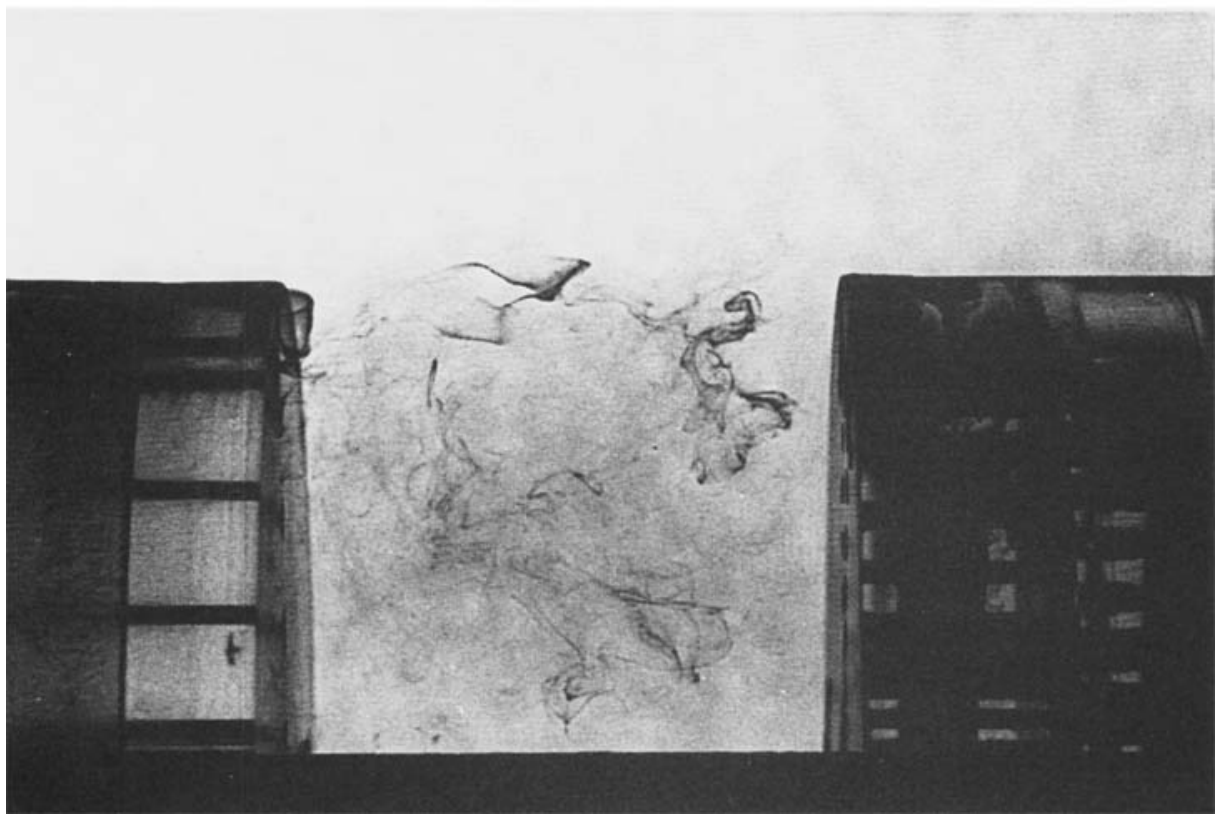

Figure 7. (a) Flow visualization of the cavity flow; $b / \theta_{0}=170$ wake mode;

(b) Close-up of the same condition.

of the momentum thickness $\theta$ of the shear layer, is given in figure 9 . As in the studies of Sarohia (1975) and Rockwell \& Knisely (1979), $\theta$ is found to grow linearly with $x / \theta_{0}$. For all cases with $b / \theta_{0} \geqslant 103$, the growth rate is fairly constant at $\mathrm{d} \theta / \mathrm{d} x=0.031$ and does not experience a sudden change in the mode-switching region. It should be noted that the cavity-shear-layer growth rate is comparable to 
(a)
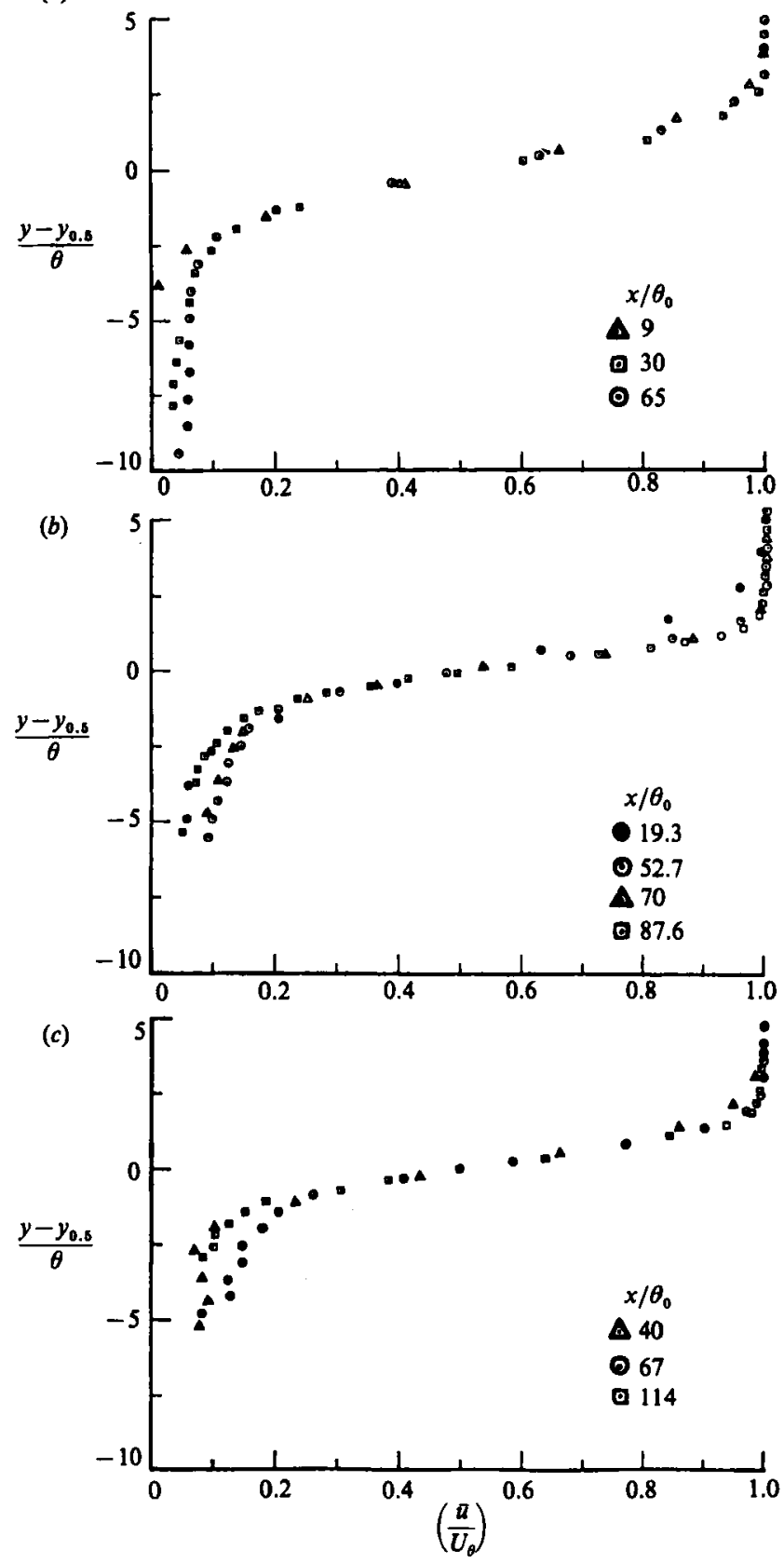

Figure 8. Non-dimensional mean streamwise velocity profiles. (a) $b / \theta_{0}=85, F=5.9 \mathrm{~Hz}$, mode II;

(b) $b / \theta_{0}=103, F=5.1 \mathrm{~Hz}$, mode II; (c) $b / \theta_{0}=130, F=6 \mathrm{~Hz}$, mode III.

that of turbulent mixing layers. For $U_{1} / U_{2}=0$, the high-Reynolds-number shear layer measured by Liepmann \& Laufer (1947) had a momentum-thickness growth rate of 0.035 .

Figure 10 presents typical profiles of the mean transverse component of the velocity, $\bar{v} / U_{\mathrm{e}}$, in the shear layer for $b / \theta_{0}=109$. Figure 11 presents the locus of $y_{0.5}$ 


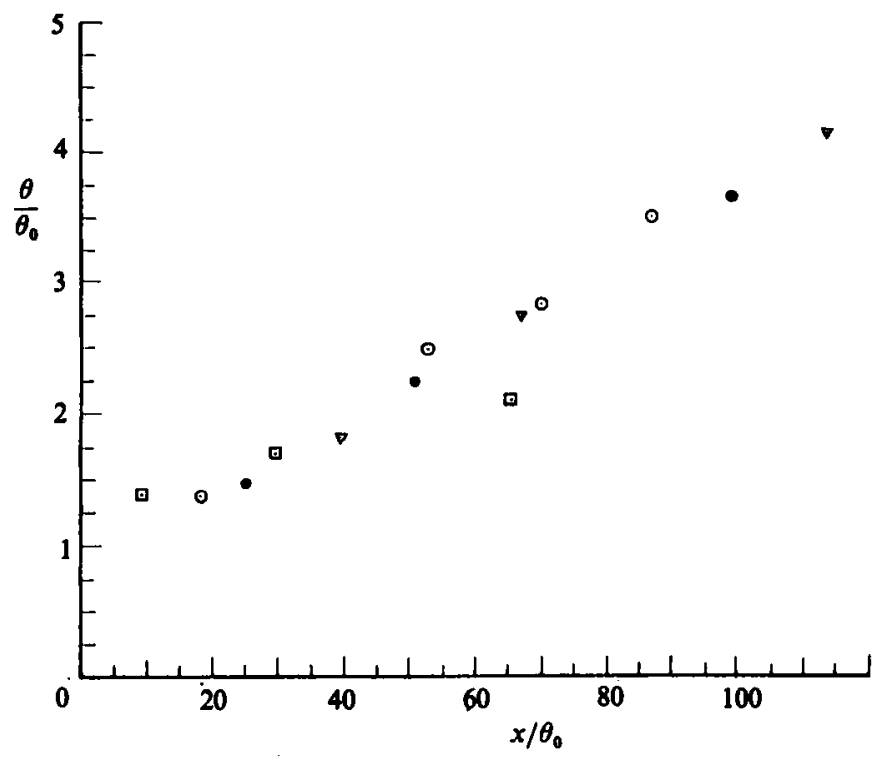

Figure 9. Non-dimensional momentum thickness growth with streamwise distance. $\square, b / \theta_{0}=85$, $F=5.9 \mathrm{~Hz}$, mode II; $\odot, b / \theta_{0}=103, F=5.1 \mathrm{~Hz}$, mode II; $\odot, b / \theta_{0}=119, F=4.9 \mathrm{~Hz}$, mode II; $\nabla, b / \theta_{0}=130, \mathrm{~F}=6 \mathrm{~Hz}$, mode III.

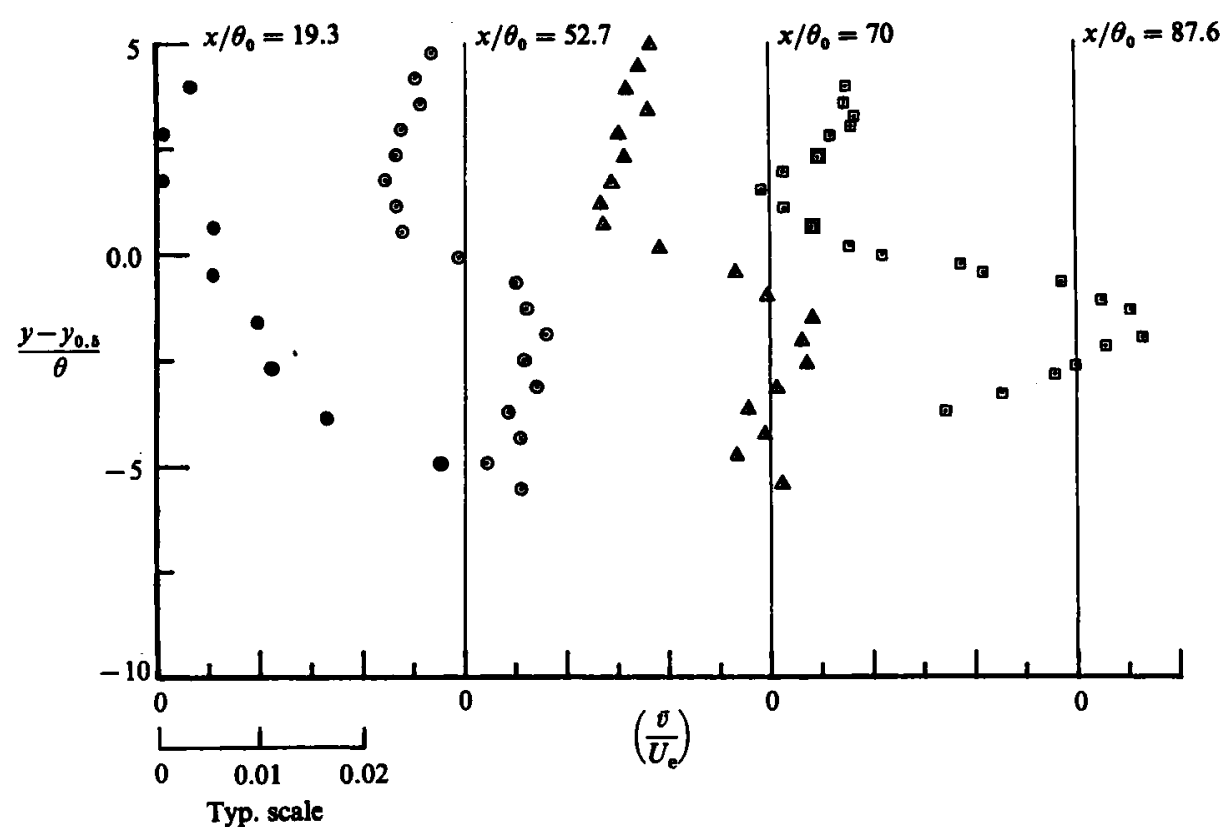

Figure 10. Non-dimensional mean transverse velocity profiles; $b / \theta_{0}=103$, $F=5.1 \mathrm{~Hz}$, mode II.

values as a function of $x / b$. As in previous studies of Browand (1966), Sarohia (1975), and Knisely (1980), the $y_{0.5}$ values were found to lie above the level of the separation edge. Depending on the stage of the cavity flow, the locus of $y_{0.5}$ approaches the $(y=0)$-line as the profile develops. The shear layer shows a stronger deflection into the cavity for $b / \theta_{0}=103$ than for $b / \theta_{0}=85$. 


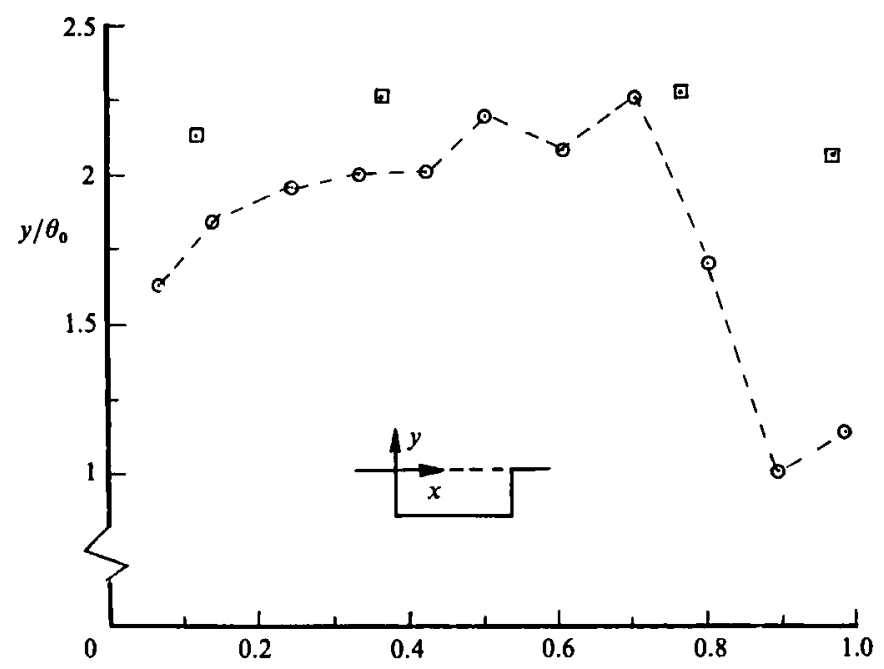

Figure 11. Locus of points $\left(y_{0.5}\right)$ where $\bar{u} / U_{\mathrm{e}}=0.5 ; \square, b / \theta_{0}=85 ; \odot, b / \theta_{0}=103$.

\subsection{Shear-stress development in the cavity shear layer}

Figure 12 depicts the streamwise evolution of the transverse profile of the shear stress for $b / \theta_{0}=85$ (mode I), $b / \theta_{0}=103$ (mode II), and $b / \theta_{0}=130$ (mode III). For large $x / \theta_{0}$, the shear-stress profiles tend to develop a second peak in the lower portion of the shear layer. A similar second peak for $\overline{-u^{\prime} v^{\prime}}$ distribution has been observed by Oster \& Wygnanski (1980) for forced turbulent shear layers. The existence of a second peak in the distribution of $\overline{u^{\prime}} / U_{\mathrm{e}}$ across the shear layers has been observed by Freymuth (1966) for free shear layers, and by Knisely (1980) for cavity shear layers. Ziada \& Rockwell (1981) showed that it can be predicted by Stuart's (1967) theoretical vortex model of a mixing layer.

Streamwise growth of the maximum shear stress for several cavity widths is shown in figure 13. Short-cavity cases, $b / \theta_{0}=85$ (mode II) and $b / \theta_{0}=103$ (mode II), show a region of exponential growth of the maximum shear stress. It should be noted that this is a broadband measurement and cannot be compared wth narrowband measurements such as those made by Freymuth (1966) in a forced mixing layer or the theoretical results of Michalke (1965). For long cavities $b / \theta_{0}>103$, the region of exponential growth is followed by a saturation region. In mode II the maximum shear stress attained is $\mathbf{0 . 0 0 8 6}$, lower than the maximum value in mode III, which is 0.013. The value of maximum shear stress for a self-similar, two-dimensional turbulent mixing layer as determined by integrating the velocity profiles presented in Liepmann \& Laufer (1947) is 0.0125 while the velocity profiles measured by Wygnanski \& Fiedler $(1970)$ give $\tau_{\max } / \rho U_{\mathrm{e}}^{2}=0.015$. Kistler \& Tan (1967) reported a maximum value of 0.012 for two-dimensional cavity shear layers. It is noteworthy that even though the thickness $\theta$ increases linearly with $x$ (figure 9) the maxima of the shear stress profiles do not have a constant value as they would in a self-similar free turbulent shear layer. 

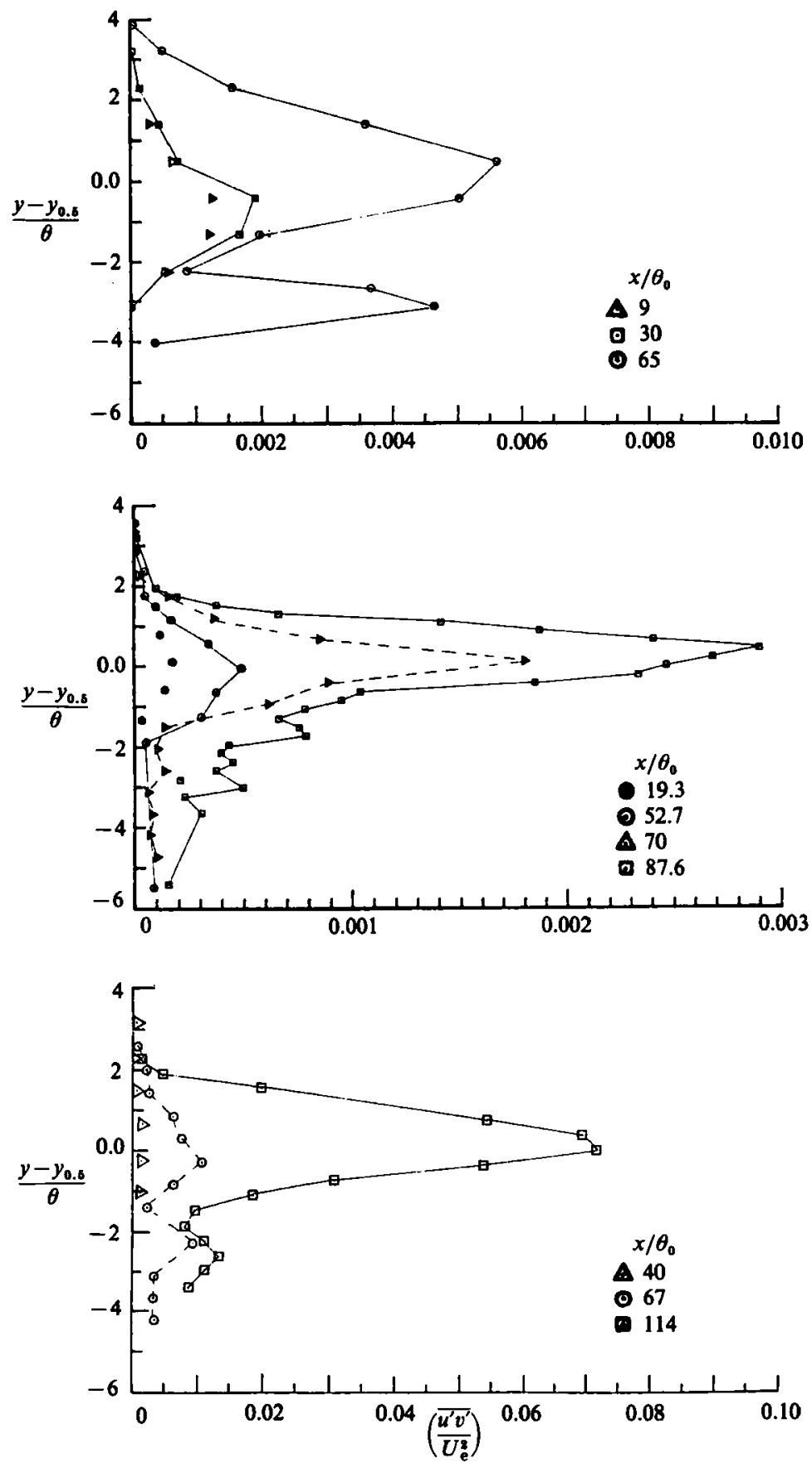

Figure 12. Reynolds stress profiles. (a) $b / \theta_{0}=85, F=5.9 \mathrm{~Hz}$, mode II; (b) $b / \theta_{0}=103$, $F=5.1 \mathrm{~Hz}$, mode II; (c) $b / \theta_{0}=130, F=6 \mathrm{~Hz}$, mode III. 

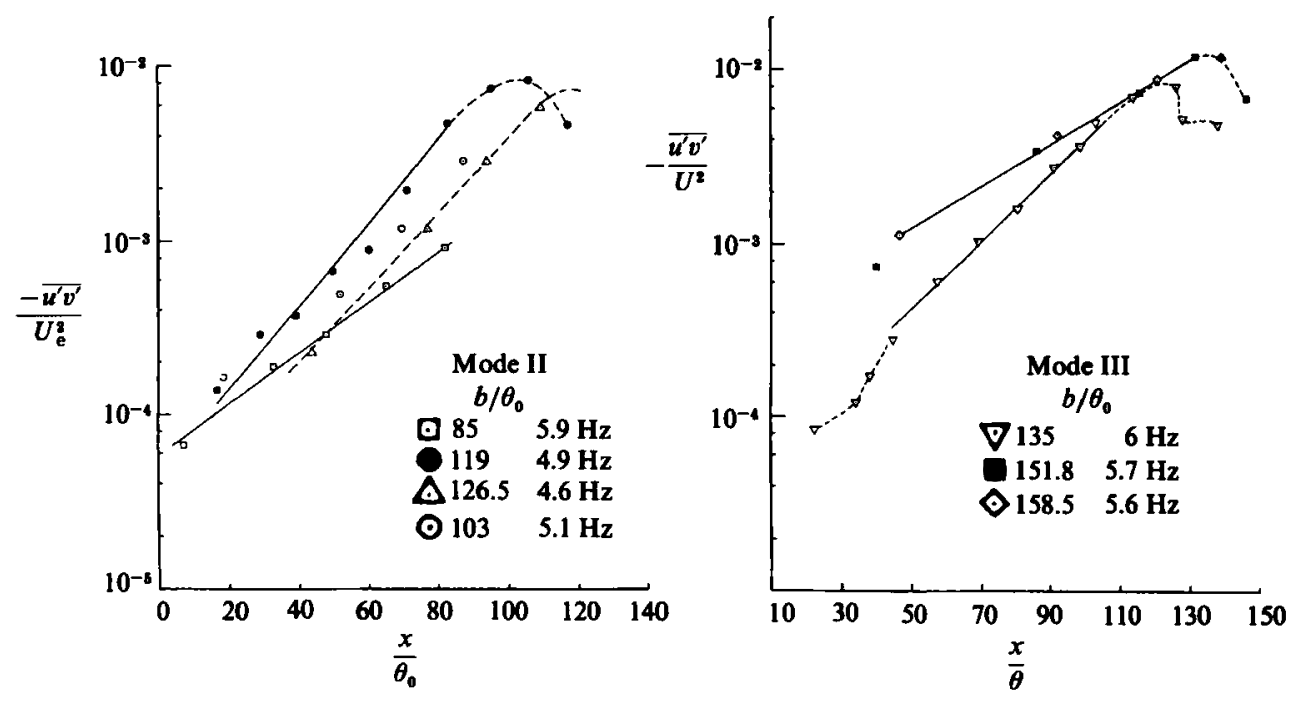

Figure 13. Growth of the maximum shear stress in the cavity shear layer with downstream distance.

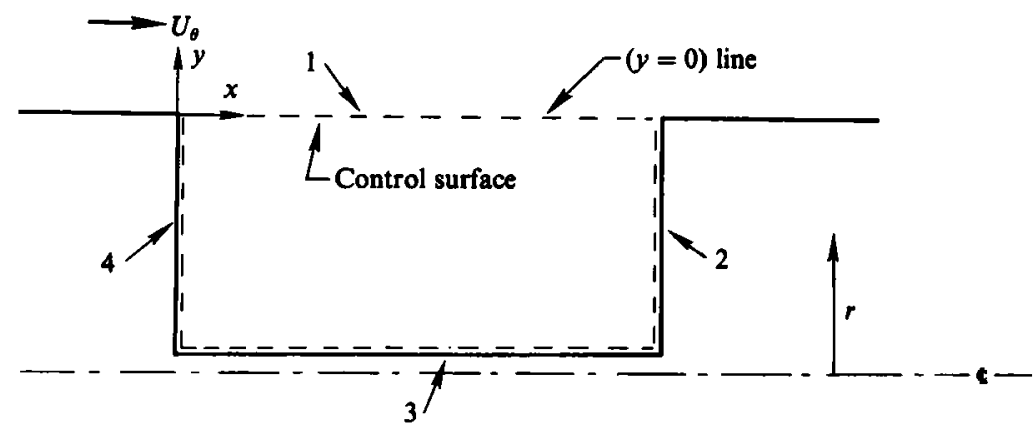

Fiqure 14. Schematics of the cavity and the control surface.

\section{The drag coefficient of the cavity}

\subsection{Momentum balance of the cavity}

Throughout this paper the term 'drag of the cavity' is used for the net force in the flow direction experienced by the walls of the cavity. Possible overall changes in the net drag of the body which might result from the cavity presence, e.g. by modification of the skin friction upstream and downstream of the cavity, are not considered. The momentum balance on the control volume shown by broken lines in figure 14 gives the relation between the drag force on the cavity walls and the momentum flux across the cavity mouth, namely

$$
D=\int_{[2]} p \mathrm{~d} A-\int_{[4]} p \mathrm{~d} A+\int_{[3]} \tau_{\mathrm{s}} \mathrm{d} A=\int_{[1]}\left(-\rho \bar{u} \bar{v}-\rho \overline{u^{\prime} v^{\prime}}+\mu \frac{\partial \bar{u}}{\partial y}\right) \mathrm{d} A,
$$

i.e. the drag $D$ may be expressed in terms of the direct forces on the cavity walls or in terms of momentum flux out of the cavity. The first two integrals give the pressure force $D_{\mathrm{p}}$ resulting from the difference of the pressures on the downstream [2] and 


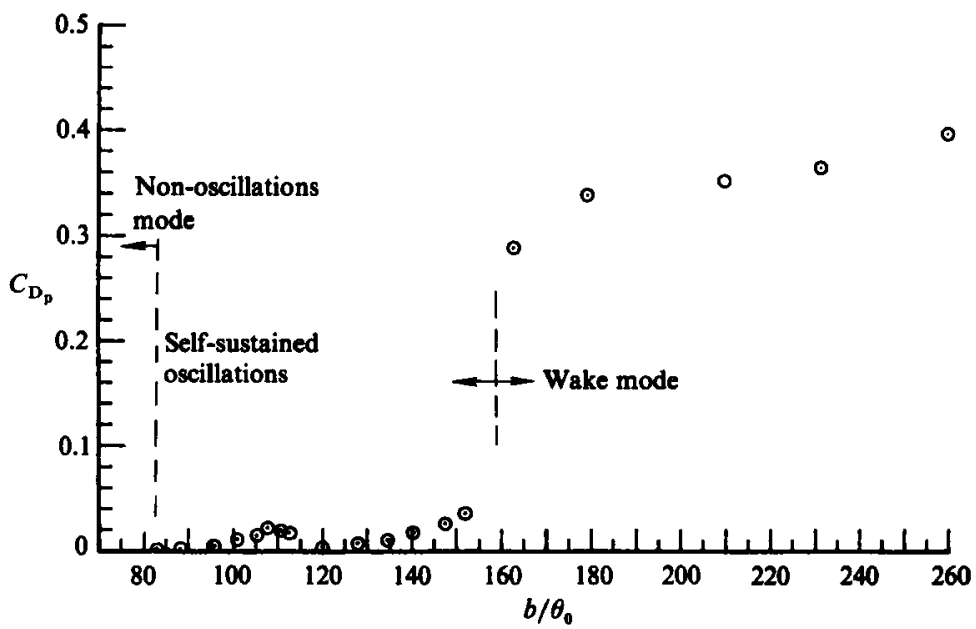

Figure 15. Variation of the axisymmetric cavity drag coefficient with non-dimensional cavity width, $b / \theta_{0}$.

upstream [4] walls, while the third one gives the force $D_{\mathrm{s}}$ on the sting due to the shear $\tau_{\mathrm{s}}$ on the sting area $A_{3}$ (or floor area in two-dimensional flow).

The integral on the right-hand side, $D_{\mathrm{M}}$, expresses the drag force in terms of the momentum flux across the area $A_{1}$. This includes the flux connected with the mean flow ( $\bar{v} \neq 0$ on $y=0$ ), the correlated fluctuating part, $-\rho \overline{u^{\prime} v^{\prime}}$, i.e. the Reynolds stress, and also the viscous stress $\mu(\partial \bar{u} / \partial y)$. The latter is negligible except in steady, laminar flow. Equation (4.1) may be represented simply by

$$
D=D_{\mathrm{p}}+D_{\mathrm{s}}=D_{\mathrm{M}}
$$

The force $D_{\mathrm{s}}$ on the sting is negligible because the dynamic pressure in the recirculating cavity flow is low (Roshko 1955) and because the sting area is small. In what follows, then, we shall use the relations

$$
D=D_{\mathrm{p}}=D_{\mathrm{M}}
$$

In non-dimensionless form, with $\frac{1}{2} \rho U_{\mathrm{e}}^{2}$ for the reference dynamic pressure and $\frac{1}{4} \pi d^{2}$, the cross-sectional area of the body, for the reference area, the drag coefficients may be expressed as

$$
C_{\mathrm{D}}=\frac{D}{\frac{1}{2} \rho U_{\mathrm{e}}^{21} \pi d^{2}}=C_{\mathrm{D}_{\mathrm{p}}}=C_{\mathrm{D}_{\mathrm{M}}} .
$$

Alternatively, using for reference the area of the mouth of the cavity, $\pi d b$, i.e. the missing surface area introduced by the cavity cutout, the force may be interpreted in terms of an equivalent average friction coefficient

$$
C_{\mathrm{F}}=\frac{D}{\frac{1}{2} \rho U_{\mathbf{e}}^{2} \pi d b}=C_{\mathrm{F}_{\mathbf{p}}}=C_{\mathrm{F}_{\mathbf{M}}} .
$$

Both coefficients give the same value of drag, but it seems natural to associate the coefficient $C_{\mathrm{D}}$ with the forces on the walls of the cavity and $C_{\mathrm{F}}$ with the momentum flux into the cavity. In what follows the drag is evaluated in both ways, i.e. from measurements of forces on the walls and from measurements of momentum flux into the cavity. 


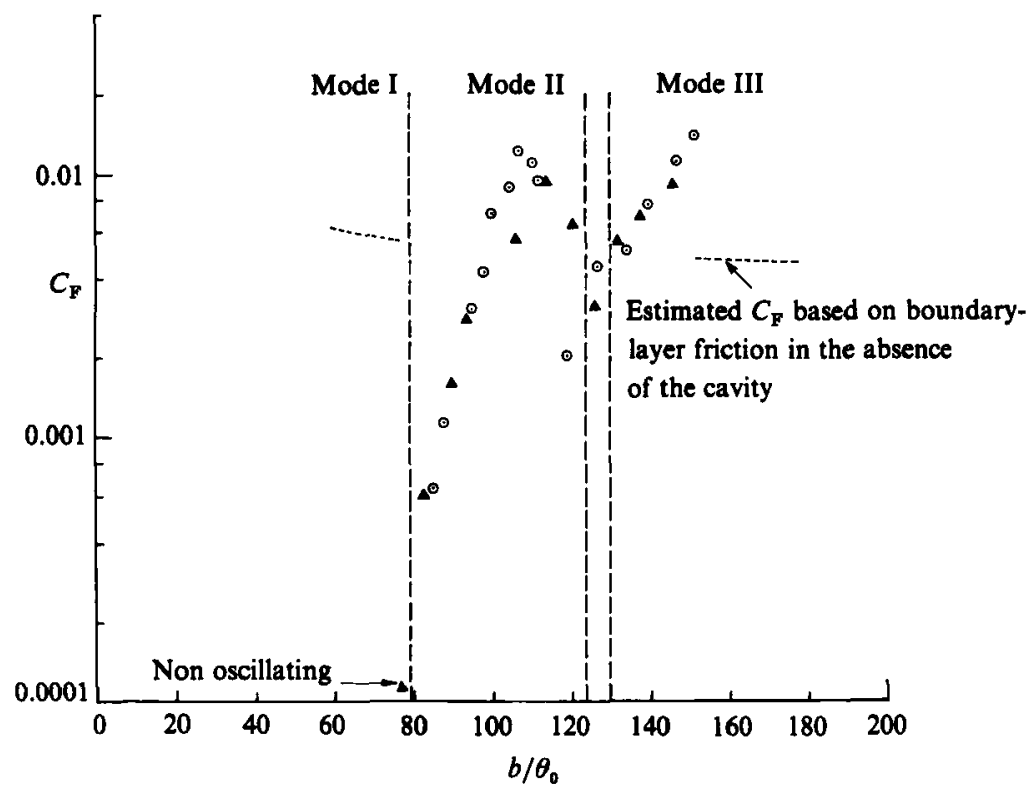

FIGURE 16. Exponential dependence of the cavity average friction coefficient on $b / \theta_{0} \odot$, pressure distribution $\left(C_{\mathbf{F p}_{\mathrm{p}}}\right) ; \mathbf{\Delta}$, momentum balance $\left(C_{\mathrm{FM}}\right)$.

\subsection{Cavity drag coefficient from pressure measurement}

The drag coefficient of the cavity $C_{\mathrm{D}_{\mathrm{p}}}$ was determined by measuring the integral of the pressure distribution over the solid walls of the cavity. Figure 15 shows the variation of $C_{\mathrm{D}_{\mathrm{g}}}$ with $b / \theta_{0}$ in the three distinct regimes of the cavity flow as defined previously. A prominent feature is that the average level of the drag coefficient is very low when the flow is in either the non-oscillating mode or the self-sustained oscillating mode but, upon the loss of self-sustained oscillation and appearance of the wake mode, it jumps to a value which is an order of magnitude higher. With further increase of the gap the drag coefficient increases monotonically and reaches a value of 0.39 at $b / \theta_{0}=250$.

In figure 16 we replot the data of figure 15 , omitting the wake mode in order to show the low drag modes on a more suitable log-linear scale, and now using $C_{\mathrm{F}}$ instead of $C_{\mathrm{D}}$ (see equations (4.4) and (4.5)). The two coefficients are related by

$$
C_{\mathrm{F}}=\frac{1}{4} \frac{d}{b} C_{\mathrm{D}}
$$

The equivalent average friction coefficient $C_{\mathrm{F}}$ in the non-oscillating mode has a typical value of $1.0 \times 10^{-4}$ at $b / \theta_{0}=77$. At the beginning of the oscillating modes (mode II in this study), its value is $6.6 \times 10^{-4}$ at $b / \theta_{0}=85$. With further increases of gap width, $C_{F}$ increases exponentially and reaches a peak value of 0.012 at $b / \theta_{0}=108$, but toward the end of mode II it decreases and reaches a low value of 0.002 at $b / \theta_{0}=120$. In mode III there is another course of exponential growth of $C_{\mathrm{F}}$ for increasing $b$ with a final value of 0.097 reached at $b / \theta_{0}=155$, where transition to the wake mode occurs. If the cavity were replaced by a solid surface, the average friction coefficient of this solid surface, based on the upstream boundary-layer-shape factor, is estimated to be 0.007 . The dotted line on figure 16 shows this estimate. It 
(a)

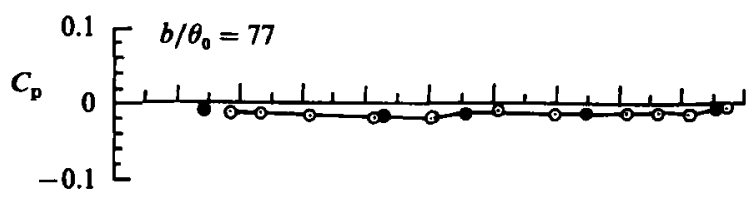

(b)

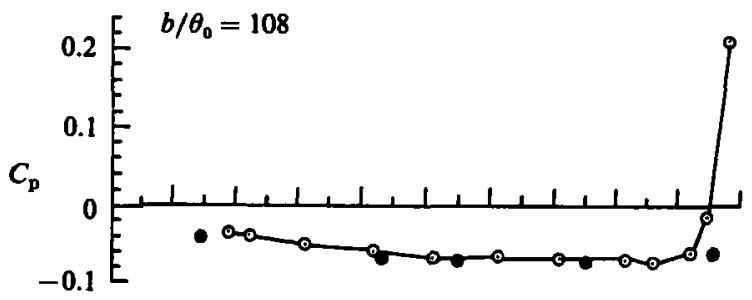

(c)

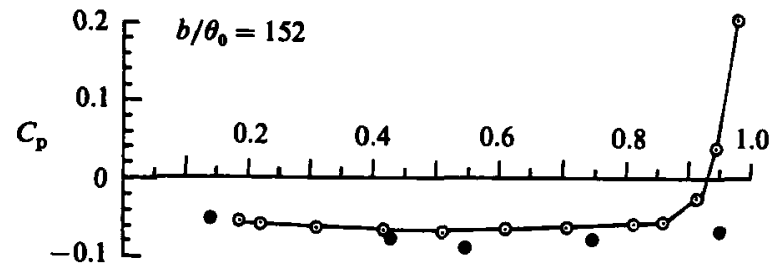

(d)

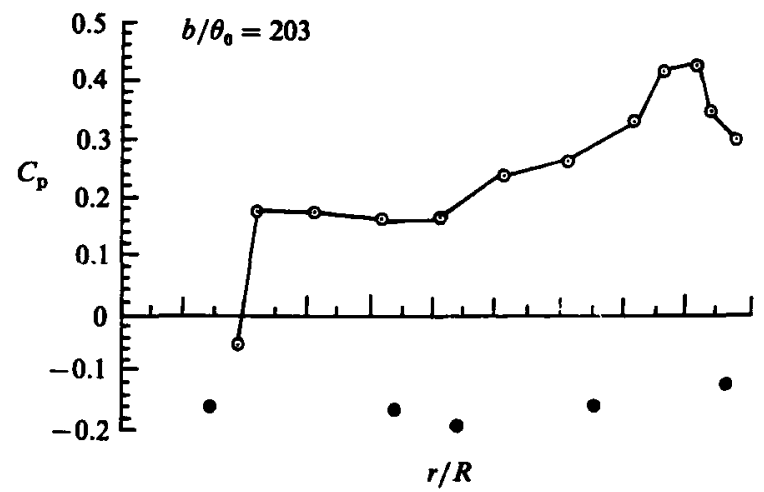

Figure 17. Pressure coefficient distribution in $(a)$ non-oscillating mode; $(b)$ mode II, $(c)$ mode III; $(d)$ the wake mode. , upstream face pressure coefficient distribution; $\odot$, downstream face pressure coefficient distribution.

seems remarkable that the measured equivalent friction drag of the open cavity can be considerably smaller than this estimate. However, one should not conclude that the addition of the cavity results in an overall reduction of drag, compared to that with a continuous surface. For example, as noted in $\$ 2$, the shape factor of the boundary layer changes from 2.5 upstream to 1.5 downstream of the cavity, suggesting that the skin friction coefficient downstream of the cavity will be at a level corresponding to a turbulent boundary layer.

\subsection{Cavity pressure distribution}

Some insight into the drag-generation mechanism can be obtained from pressure distributions along the solid surfaces of the cavity.

Figure 17 presents the plot of the pressure coefficient $C_{\mathrm{p}}=\left(P-P_{\mathrm{e}}\right) / \frac{1}{2} \rho U_{\mathrm{e}}^{2}$, along a 


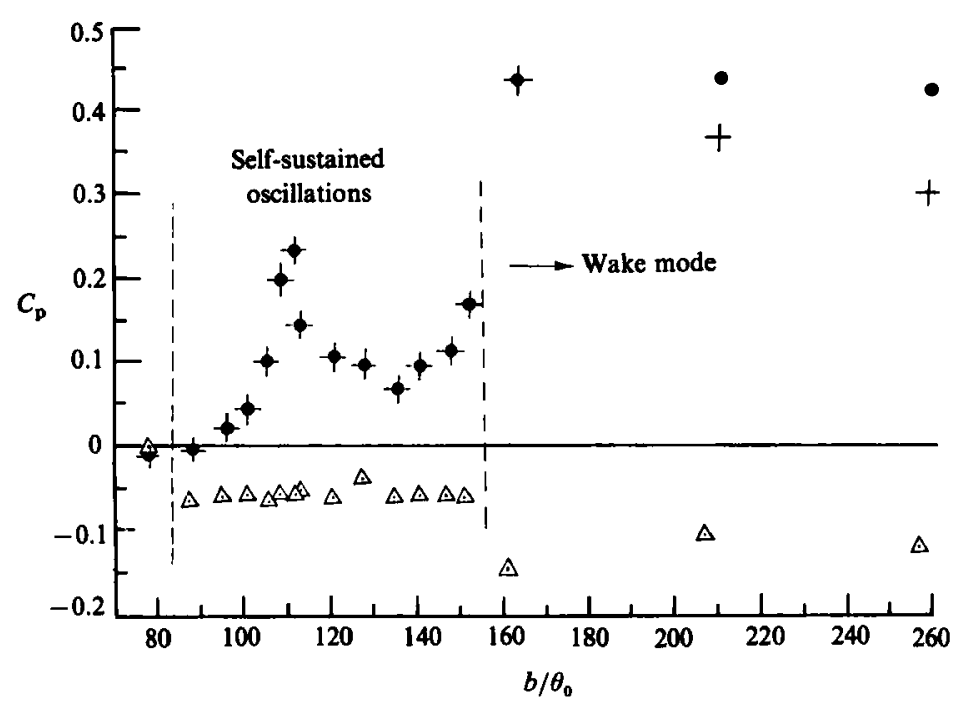

FIoURE 18. Variation of maximum pressure coefficient with $b / \theta_{0} ; \Delta, C_{\mathrm{p}}$ upstream corner; ,$+ C_{\mathrm{p}}$ downstream corner;,$C_{\mathrm{pmax}}$ downstream.

body radius at the upstream and downstream faces of the cavity, for several selected values of $b / \theta_{0}$. In the non-oscillating regime, the cavity pressure coefficient is slightly negative, $C_{\mathbf{p}}<0$, and nearly uniform; i.e. no significant differences are observed between the upstream- and downstream-face pressure distributions, which correspond to closed and open symbols, respectively.

Figure $17(b, c)$ shows typical cavity-pressure-coefficient distributions for modes II and III of oscillation. Again the pressure coefficients are negative and nearly equal on upstream and downstream walls but now there is a positive pressure peak near the downstream corner, with the maximum value $\left(C_{\mathbf{p}_{\max }}\right)$ just at the corner. Thus the contribution to the total drag from the deeper portions of the cavity walls cancel each other, leaving only the portion near the edge of the downstream face, with $C_{\mathrm{p}}>0$, as the main contributor to the cavity drag.

For the wake mode a typical pressure coefficient distribution is shown in figure $17(d)$. Two main features distinguish the wake mode from the oscillating mode. First, the entire downstream face of the cavity experiences positive pressure, i.e. $C_{\mathrm{p}}>\mathbf{0}$. Also, the level of maximum pressure is higher compared to that of the oscillating mode. Secondly, the average upstream face pressure is $40 \%$ lower than in the oscillating case. Thus the large drag in this mode is due to the large difference in pressures on the downstream and upstream faces and to the broader distribution of the pressure difference.

Figure 18 shows how the maximum pressure $\left(C_{\mathrm{p}_{\max }}\right)$ varies with $b / \theta_{0}$. In the regime of self-sustained oscillation it occurs at the downstream corner, as indicated by the overlap of the symbols. The appearance of the wake mode is marked by a sudden increase in the value of $C_{\mathrm{p} \max }$ from +0.17 to +0.43 at $b / \theta_{0} \approx 162$ at the downstream corner. These changes correspond to those in the drag coefficient in figure 15. In the wake mode the pressure at the corner decreases strongly with increasing $b$, as the position of $C_{\mathrm{p}_{\max }}$ moves inward on the rear face. $C_{\mathrm{p}_{\max }}$ itself decreases only slightly. This is consistent with a picture in which the front body is trying to form a closing wake which the rear body intercepts further and further downstream. 

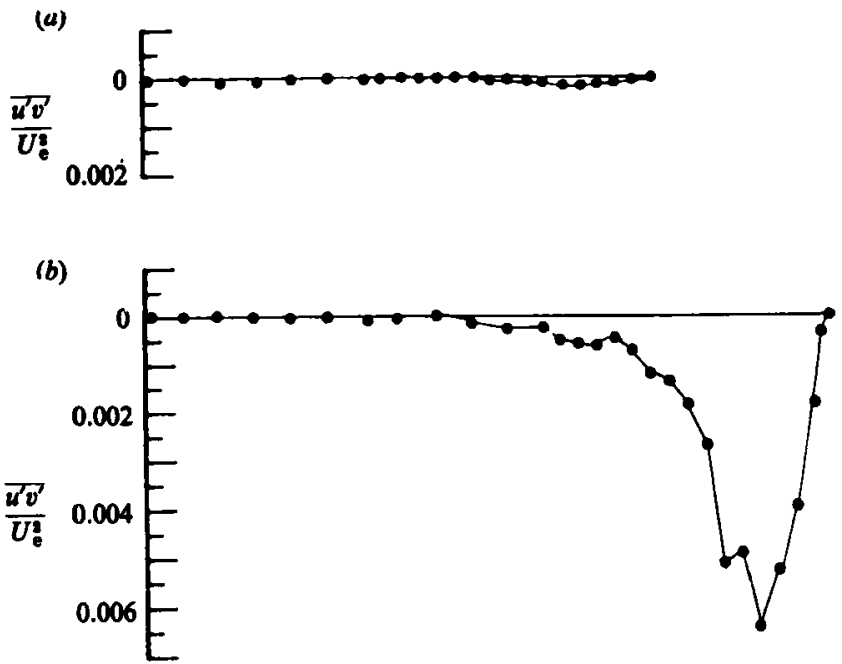

(c)

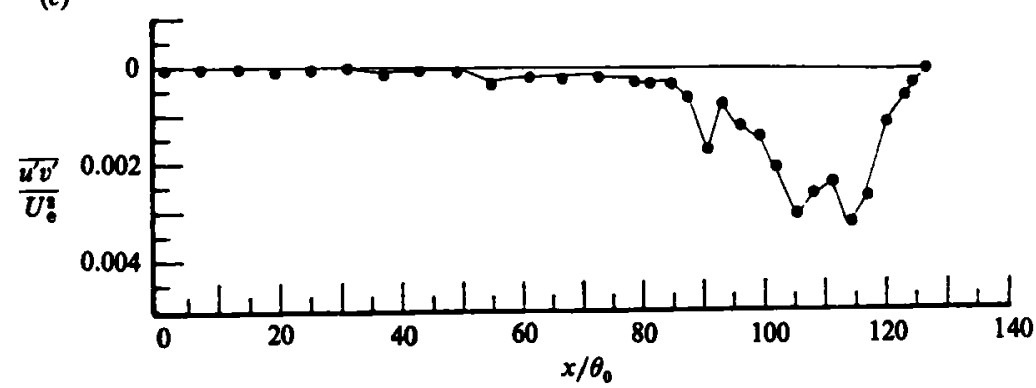

(d)

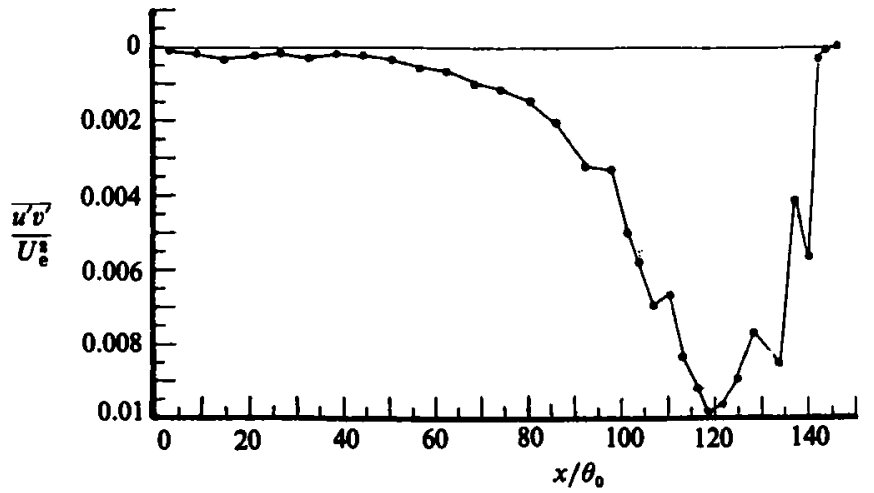

FiquRe 19. Distribution of the Reynolds stress $\left(\overline{u^{\prime} v^{\prime}} / U_{\mathrm{e}}^{2}\right)$ along $(y=0)$-line. (a) Mode II, $b / \theta_{0}=83$; (b) mode II, $b / \theta_{0}=112.5 ;(c)$ mode II; $b / \theta_{0}=126$. (d) mode III, $b / \theta_{0}=146$.

It is noteworthy that in the self-sustained modes the location of $C_{\mathrm{p}_{\max }}$ is fixed in the vicinity of the upstream corner. Displacement of this point toward the interior of the cavity begins only when the cavity loses the self-sustained oscillation to the wake mode. In the self-sustained oscillating mode the shear layer locks on to the downstream corner. This point is discussed more fully in $\$ 5$. 


\subsection{Cavity drag coefficient from momentum balance}

$C_{\mathrm{F}_{\mathrm{M}}}$ can be obtained by integrating the turbulent shear stress and mean momentum flux over the mouth of the cavity (equation (4.1)). Both turbulent and mean momentum-transfer terms contribute to the net cavity drag but the viscous term is neglected. We were interested to determine the relative importance of these terms in different stages of the oscillations. LDV technique was used to measure the $x$ - and $y$-components of the velocity vector, and to determine the momentum flux terms $\overline{u v}$ and $\overline{u^{\prime} v^{\prime}}$. For each case, from 20 to 30 measurement points were selected along the $(y=0)$-line. Smaller spacing was used close to the downstream corner. It was difficult and time consuming to obtain reliable measurements in the wake mode due to the flow-field asymmetry of that mode. Thus, our evaluation of the $\bar{u} \bar{v} / U_{\mathrm{e}}^{2}$ and $\overline{u^{\prime} v^{\prime}} / U_{\mathrm{e}}^{2}$ terms in the momentum integral is given only for the self-sustained oscillating modes of the cavity flow.

The variation of $C_{\mathrm{F}_{\mathrm{M}}}$ with $b / \theta_{0}$, determined by integration of $\bar{u} \bar{v} / U_{\mathrm{e}}^{2}$ and $\overline{u^{\prime} v^{\prime}} / U_{\mathrm{e}}^{2}$ along the $(y=0)$-line, is plotted in figure 19 where it may be compared with the values $C_{\mathrm{FP}}$ determined from pressure measurements. This independent determination confirms the exponential growth of $C_{\mathrm{F}}$ in the oscillating modes; in fact, the absolute values from both methods agree fairly well, except for $b / \theta_{0}=121$, which is in the transition region between modes II and III, and $b / \theta_{0}=107$, which is near the point of maximum amplitude in mode II. It seems clear from the trend of the data that $C_{\mathrm{FP}_{\mathrm{P}}}$ are the more accurate values. In fact, $C_{\mathrm{F}_{\mathrm{M}}}$ is the more difficult measurement because it involves the contribution of $\bar{u} \bar{v}$, whose integral value over the line $(y=0)$ is a relatively small difference of two large contributions, as may be seen in figure 20 .

\subsection{Distribution of $\overline{u^{\prime} v^{\prime}}$ and $\bar{u} \bar{v}$ along the $(y=0)$-line}

Figure $19(a-c)$ shows the distribution of Reynolds stress, $\overline{u^{\prime} v^{\prime}} / U_{\mathrm{e}}^{2}$ for mode II. It increases along the line $(y=0)$, reaches a peak and goes to zero steeply as the flow approaches the downstream corner. The largest value of this peak in mode II, occurs for the cavity with $b / \theta_{0}=112.5$, which corresponds to the maximum drag case. In the transition region $\left(b / \theta_{0}=126\right)$ the peak value of the shear stress decreases to 0.003 .

Figure $19(d)$ shows an example of the distribution of Reynolds stress in mode III, for $b / \theta_{0}=146$. The broad peak is typical for this mode.

The $\bar{u} \bar{v} / U_{\mathrm{e}}^{2}$ term was evaluated along the $(y=0)$-line, using the independent measurements of $\bar{u} / U_{\mathrm{e}}$ and $\bar{v} / U_{\mathrm{e}}$; the results are shown in figure $20(a-d)$. A typical distribution of $\bar{u} \bar{v} / U_{\mathrm{e}}^{2}$ shows a wide region of positive values for $\bar{u} \bar{v} / U_{\mathrm{e}}^{2}$, followed by a negative peak close to the downstream corner. The integrated value

$$
\int_{0}^{b} \frac{\overline{u v}}{U_{\mathbf{e}}^{2}} \mathrm{~d} x
$$

is always negative, i.e. it makes a positive contribution to the drag of the cavity.

For convenience, the following conventions are introduced. The contribution of the $\bar{u} \bar{v}$ term to the equivalent average friction coefficient is

$$
C_{\mathbf{F} \bar{u} \bar{v}}=-\frac{2}{b} \int_{0}^{b} \frac{\bar{u} \bar{v}}{U_{\mathrm{e}}^{2}} \mathrm{~d} x .
$$

The contribution of the $\overline{u^{\prime} v^{\prime}}$ term to the equivalent average friction coefficient is

$$
C_{\mathrm{F} \bar{u}^{\prime} v^{\prime}}=-\frac{2}{b} \int_{0}^{b} \frac{\overline{u^{\prime} v^{\prime}}}{U_{\mathrm{e}}^{2}} \mathrm{~d} x .
$$



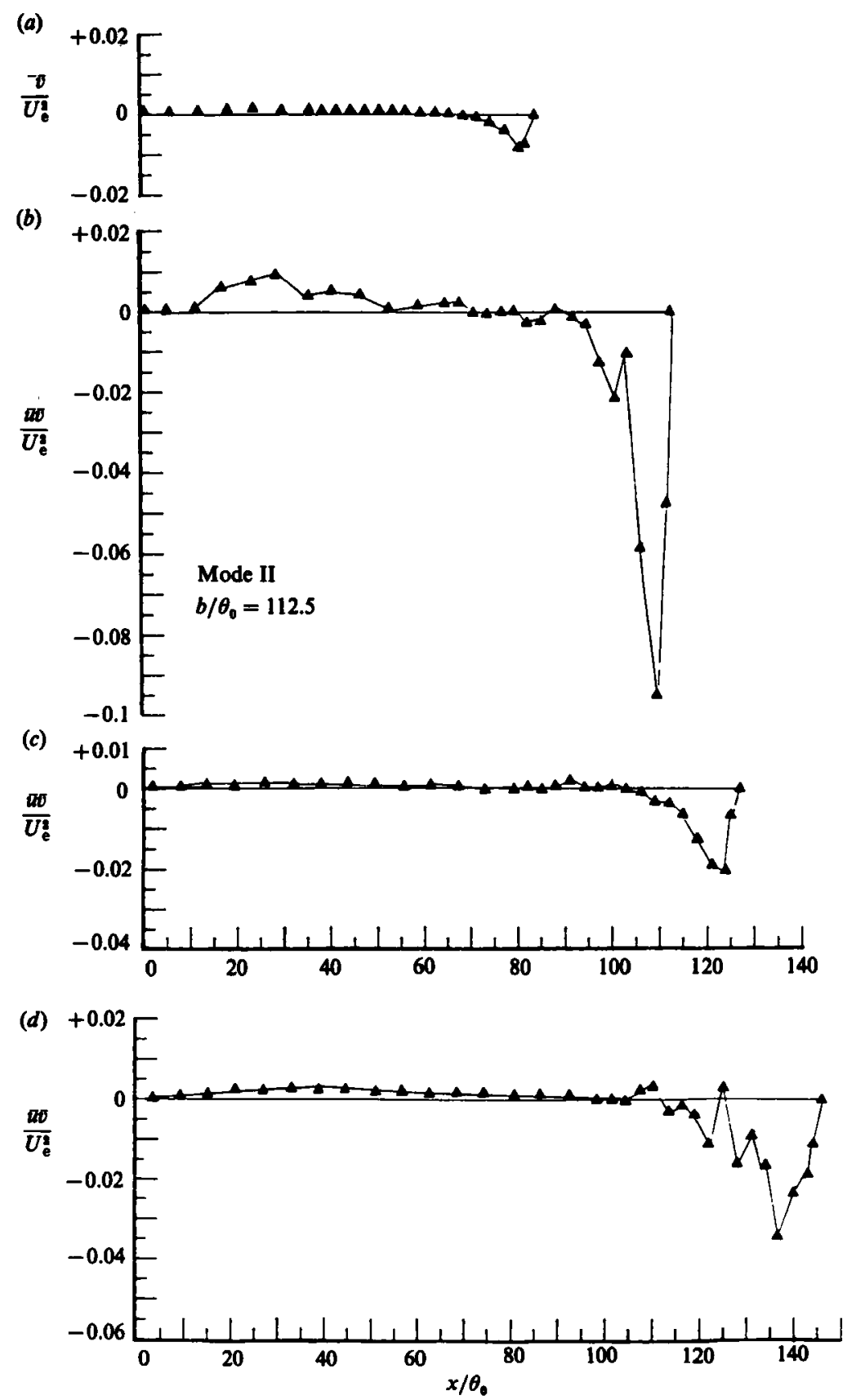

FigURE 20. Distribution of the mean momentum transfer $\left(\bar{u} \bar{v} / U_{\mathrm{e}}^{2}\right)$ along $(y=0)$-line. (a) Mode II, $b=\theta_{0}=83 ;(b)$ mode II, $b=\theta_{0}=112.5 ;(c)$ mode II, $b=\theta_{0}=126 ;(d)$ mode III, $b=\theta_{0}=146$.

The net equivalent friction coefficient is

$$
C_{\mathbf{F}}=C_{\mathbf{F}_{\bar{u} v}}+C_{\mathbf{F}_{\bar{u} v^{v}}} .
$$

A comparison of these contributions to the equivalent friction coefficient is given in figure 21 .

This figure shows that in mode II, especially in the beginning stages, $C_{\mathrm{F}_{\bar{u} \bar{v}}}$ 


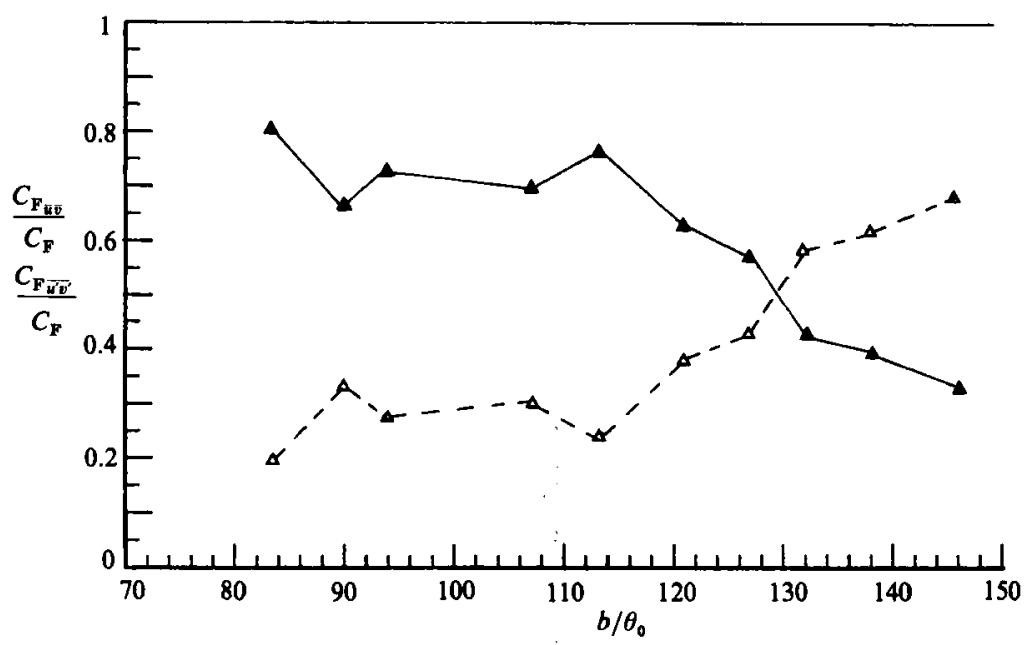

Figure 21. $\triangle$, Reynolds stress contribution to the net cavity drag; $\Delta, \bar{u} \bar{v} / U_{\mathrm{e}}^{2}$ term contribution to the net cavity drag.

contributes up to $80 \%$ of the total drag coefficient. As $b / \theta_{0}$ increases, the contribution of $C_{\mathrm{F}_{\bar{u}, v^{\prime}}}$ becomes more important but it never exceeds that of $C_{\mathrm{F}_{\bar{u} \bar{v}}}$. Mode III shows a dominant role for $C_{\mathrm{F}_{\bar{u} \bar{v}}}$; immediately after transition, $C_{\mathrm{F}_{\overline{u^{\prime} v^{\prime}}}}$ constitutes $60 \%$ of $C_{\mathrm{F}}$ and increases to $70 \%$ at the critical gap $\left(b / \theta_{0}=146\right)$. The message of figure 21 is clear; assuming the Reynolds stress $\overline{u^{\prime} v^{\prime}} / U_{\mathrm{e}}^{2}$ to be the only means of momentum transfer across the surface $y=0$ may result in a $30-80 \%$ underestimation of the cavity drag, depending on cavity mode and length. For example, Kistler \& Tan (1967) used a combined approach similar to ours to determine the drag of a cavity (with turbulent upstream boundary layer). His estimate of the drag based on the distribution of $\overline{u^{\prime} v^{\prime}}$ along the cavity mouth, from hot-wire measurements, was $60 \%$ lower than the value obtained from his measurement of the pressure distribution. The measurements did not include $\bar{u} \bar{v}$. (Of course, the measurement of $\bar{v}$ in these flows is difficult if not impossible by conventional hot-wire techniques.)

It should be noted in passing that an alternative method for evaluating the drag would be to locate the zero or 'dividing' streamline between the upstream and downstream corners. This line will be curved, in general. There will still be an $x$-component of a (different) Reynolds stress along this surface but the mean momentum flux across it will, of course, be zero. But now there will be a streamwise component of the pressure force acting on the control surface. Clearly this would be a more difficult determination than the one used here. (Within linearized theory the shape of the zero streamline can be determined from

$$
\left.\int_{0}^{x} \bar{v} \mathrm{~d} x .\right)
$$

\subsection{Distributions of $\bar{u}$ and $\bar{v}$ along $y=0$}

A typical distribution of $\bar{u} / U_{\mathbf{e}}$ (figure 22) shows an increase from zero at $x=0$ to a peak value, followed by a rapid decrease to zero at $x=b$.

In figure $23(a-d)$, a sequence of $\bar{v} / U_{\mathrm{e}}$ distributions for several selected values of $b / \theta_{0}$ in mode II is given. Each figure is accompanied by its corresponding drag coefficient. A common feature of all $\bar{v} / U_{\mathrm{e}}$ distributions is the existence of a narrow 
(a)
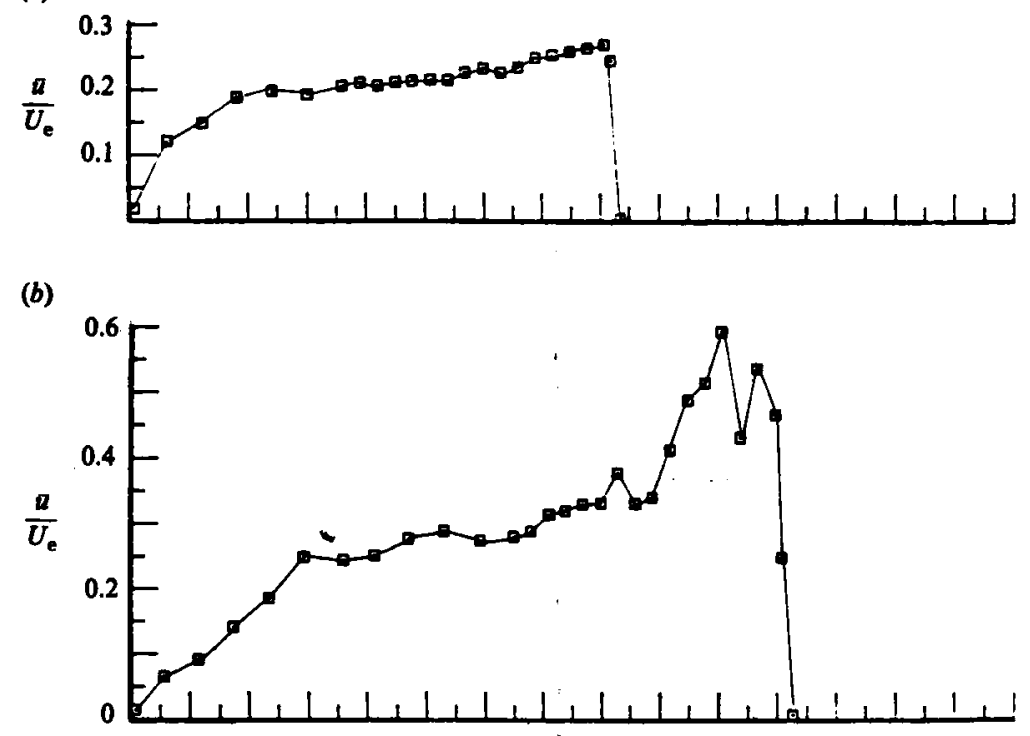

(c)

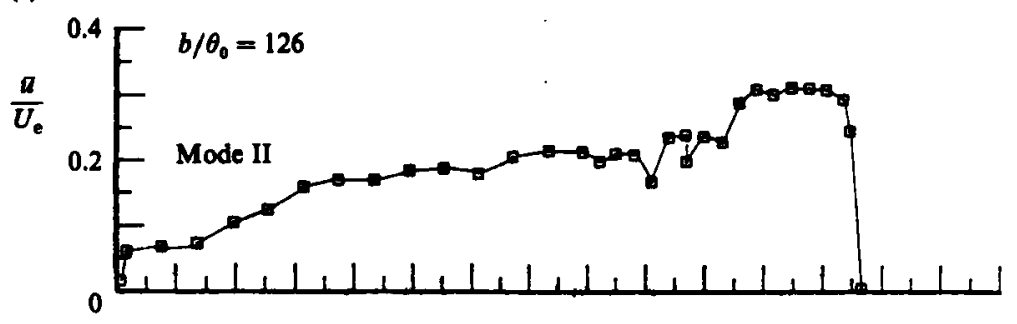

(d)

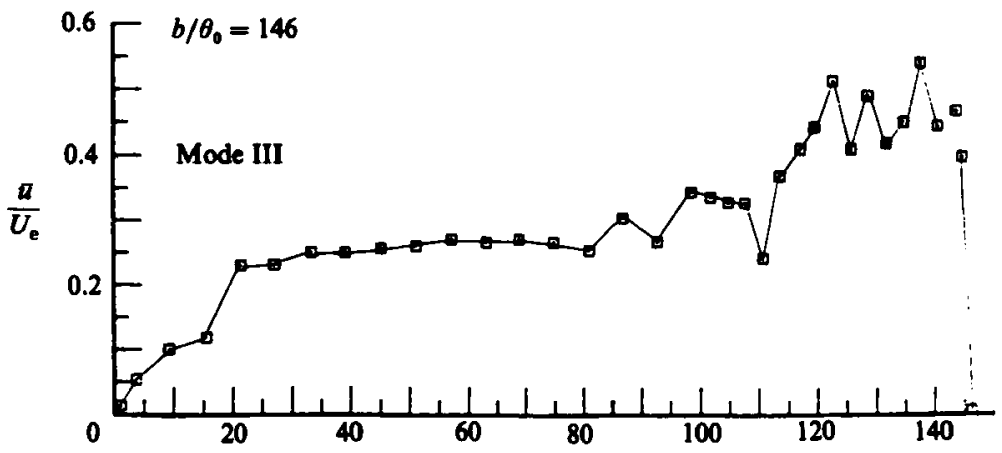

Figure 22. Distribution of the mean streamwise velocity $\left(\bar{u} / U_{\mathrm{e}}\right)$ along $(y=0)$-line. (a) $C_{\mathrm{D}}=0.8 \times 10^{-3}, b / \theta_{0}=83 ;(b)$ mode II, $b / \theta_{0}=112.5 ;(c)$ mode II, $b / \theta_{0}=126 ;(d)$ mode III. $b / \theta_{0}=146$.

region of inflow to the cavity $(\bar{v}<0)$ close to the downstream corner. The negative $\bar{v}$ region is balanced, upstream, by a broader region of outflow $(\bar{v}>0)$. The peak value of $=v$ increases as $b / \theta_{0}$ increases and has a maximum for the cavity for which $b / \theta_{0}=112.5$; this is the case for which $C_{\mathrm{F}}$ has its maximum value in mode II. This peak eventually decreases toward the end of mode II. In mode III the negative $\bar{v}$ region becomes slightly broader (figure $23 d$ ). 
(a)

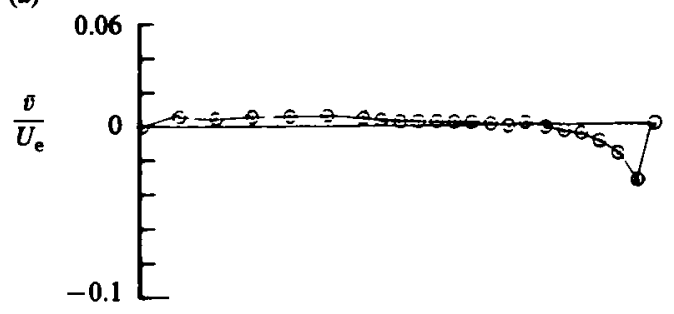

(b)

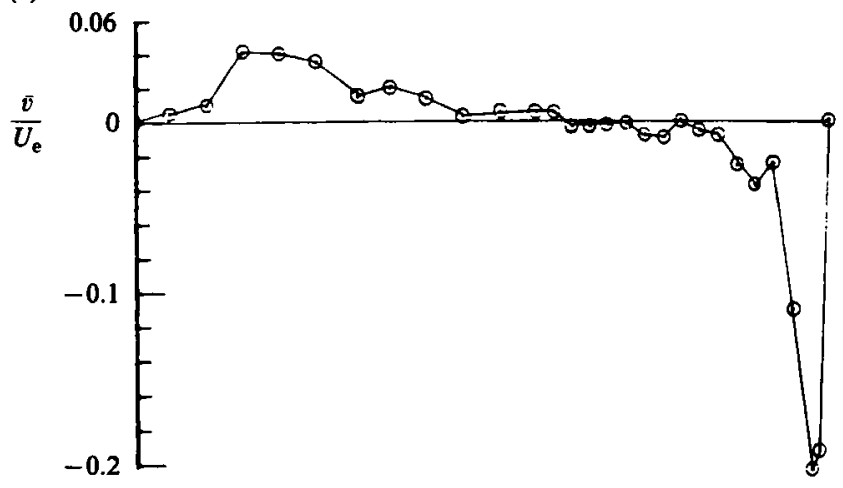

(c)

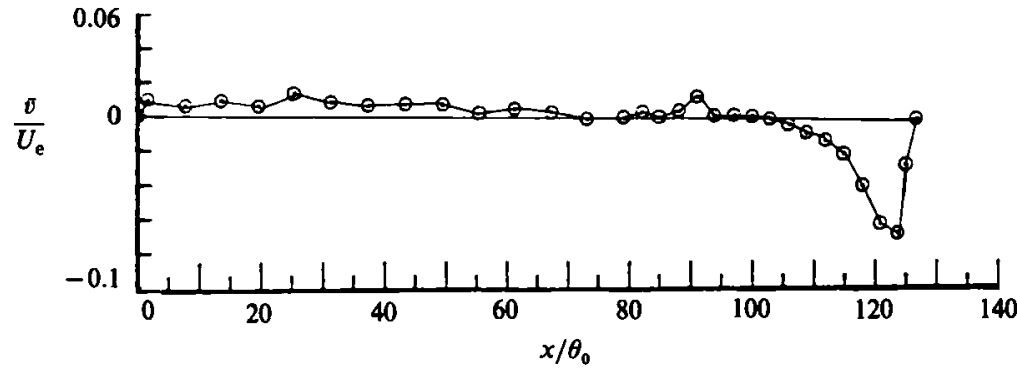

(d)

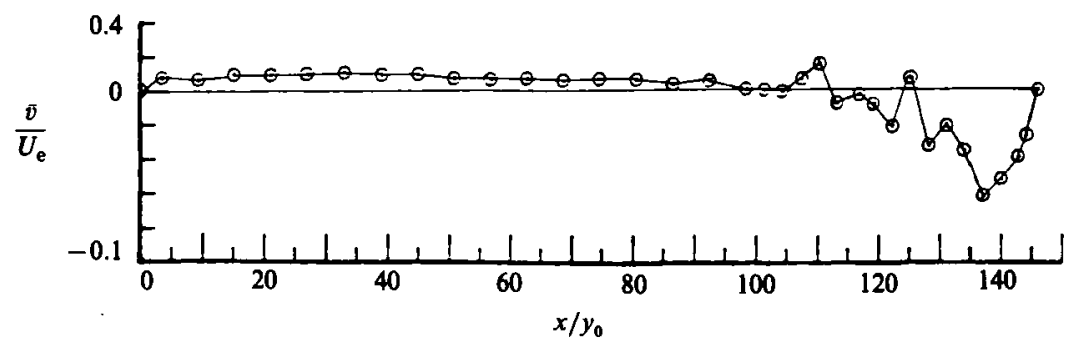

Figure 23. Distribution of the mean transverse velocity $\left(\bar{v} / U_{\mathrm{e}}\right)$ along $(y=0)$-line. $(a) b / \theta_{0}=83$, $F=6 \mathrm{~Hz}$, mode II; $(b) b / \theta_{0}=112.5, F=5 \mathrm{~Hz}$, mode II; (c) $b / \theta_{0}=126, F=4.6 \mathrm{~Hz}$, mode II; (d) $b / \theta_{0}=146, F=5.8 \mathrm{~Hz}$, mode III.

The recirculating (vortex) flow inside the cavity is related to the distribution of $\bar{v}$ along $y=0$, which can (approximately) be interpreted as a distribution of sinks and sources for the interior flow. Thus, increasing amplitude of the $v(x, 0)$ distribution results in increasing strength of the cavity vortex. For example, the strength of the vortex increases during mode II as $b / \theta_{0}$ increases, thus the strongest vortex appears 
at the drag-maximum case, i.e. at $b / \theta_{0}=112.5, F=5 \mathrm{~Hz}$. The cavity vortex becomes weaker during transition and recovers its strength during mode III. These trends were observed with dye flow visualization and with direct measurements, not shown here, of mean flow velocities in the cavity (Gharib 1983).

\section{Discussion of flow characteristics}

The cavity shear layer in self-sustained oscillation is different from a free shear layer, either one that is fully turbulent or even one that is in its initial stages of instability. A principal difference, of course, is the strong phase coherence of the oscillations in the cavity shear layer as compared to a lack of coherence in free, unforced shear layers. For cavity oscillations the spectrum has a sharp peak at the particular mode frequency while in a free, unforced shear layer even the KelvinHelmholtz instability has a broadband spectrum (Browand 1966) around the 'natural' frequency. The linear growth of the momentum thickness in the cavity shear layer (figure 9) would seem to suggest that the development is self similar, like that in a turbulent free shear layer, but self-similarity is not consistent with the non-constant value of the maximum shear stress. In fact, the latter increases exponentially, in concert with the amplifying oscillation.

It is also of interest to compare the stability 'windows' for the cavity oscillation with those of a free shear layer. From the work of Michalke (1965) and Freymuth (1966) the theoretical window for free shear layers is $12.6<\lambda / \theta_{0}<\infty$, with maximum amplification at $\lambda / \theta_{0}=30.4$. In practice, Freymuth could excite disturbances only in the interval $18<\lambda / \theta_{0}<\infty$. In the cavity flow (figure 4) the mode II oscillations $\left(\lambda=\frac{1}{2} b\right)$ occur for $42<\lambda / \theta_{0}<65$; mode III $\left(\lambda=\frac{1}{3} b\right)$ also begins at $\lambda / \theta_{0}=42$ but, in our experiment, its range was not completed before it was supplanted by the wake mode. Thus, for wavelengths to which the free shear layer is most receptive, the cavity shear layer does not respond at all. The cavity shear layer response begins at relatively long wavelengths and increases with increasing wavelength (due to the increasingly amplified feedback) until supplanted by the next higher mode.

From the comparisons in the preceding paragraphs, it is clear that the oscillating shear layer in the cavity is fundamentally different from a free shear layer. This difference, of course, is attributable to coupling between the upstream and downstream edges of the cavity. It also raises the question as to whether a truly 'free' shear layer (oscillating or turbulent) exists, since there will always be an influence at the separation point from the downstream large structure (waves or vortices) and eventually the latter will be interacting with some geometrical constraint (Dimotakis \& Brown 1976). For example, for a reattaching shear layer the wall is a constraint. The constraint is usually not so strong as that at the downstream corner in the cavity but will nevertheless have some influence on developments upstream and thus may have a roll in the overall flow dynamics. Whether in such circumstances the shear layer is 'free' or is part of a system in which upstream coupling plays a role may depend on a threshold value for the amplitude of the fluctuation at the downstream edge.

The flows studied here oscillated in pure modes up to the values of gap width, $b / r=1.3$, at which the wake mode began. The corresponding maximum value of $b / \theta_{0}$ was 160 . It is of interest to ask what would happen if the wake mode were delayed by keeping $b / r<1.3$, but increasing $b / \theta_{0}$ indefinitely by reducing $\theta_{0}$, say by increasing $U$ or reducing $\nu$ (thus $U \theta_{0} / \nu$ would increase). The cavity mode number 


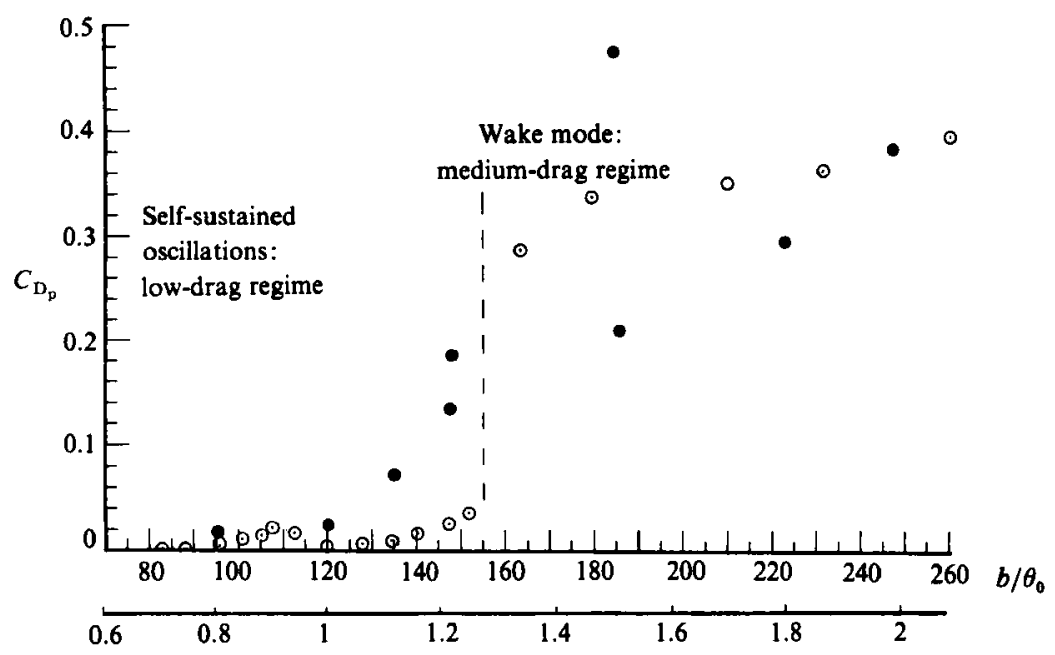

Figure 24. Variation of the cavity drag coefficient with $b / R ; \odot$, present study; $\odot$, Koenig \& Roshko (1985) optimum drag coefficient of disk-cylinder combination.

would, potentially, increase but at the same time the tendency toward 'turbulent' flow would aso increase. For example, a free shear layer is 'fully turbulent' by $x / \theta_{0} \approx 10^{3}$ (Bradshaw 1966). We surmise that for $\theta_{0} \ll b$ such that $N \gg 1$ the instability waves in the initial part of the shear layer would become uncoupled from the downstream corner and the cavity would continue to select a low-mode oscillation. The development of the initial portion would be similar to that of a free shear layer but modulated by a low-mode cavity wave and possibly exhibiting the 'collective interactions' and enhanced growth rate described by Ho \& Huang (1982) and Oster \& Wygnanski (1982). This conjecture is supported by the observations of Sarohia \& Massier (1977), who found low-mode cavity oscillations in an axisymmetric configuration similar to ours but at much higher Reynolds number and with the boundary layer turbulent upstream of the cavity.

In the preceding we have introduced the terms 'self-sustained oscillation modes' and 'wake mode' as descriptive of the different flow regimes we have studied. The appearance of the wake mode is accompanied by a large increase of drag. Such dramatic increases of drag and corresponding changes of cavity pressure field had previously been observed by Roshko (1955), Fox (1968), Koenig \& Roshko (1985), but the nature of the corresponding change of flow, especially the role of the self-sustained oscillations in the low-drag regime, had not been well understood. In this section we discuss further the flow characteristics of these regimes.

In their study of coaxial flow over a combination of disk and cylinder separated by a gap, Koenig \& Roshko (1985) defined two 'optical' flow regimes based on the level of drag of the combination, namely 'low' and 'medium' drag regimes (figure 24). The medium drag is significantly lower than the drag that exists when the bodies are well separated and the downstream body has little influence on the upstream body, but in the low-drag regime it is about an order of magnitude even lower. The separated shear layer is relatively thin, though turbulent, and, in 'optimal' flows, joins smoothly onto the shoulder of the downstream cylinder, while in the mediumdrag regime the shear layer develops oscillations on a much larger scale. Their study suggests that their low-drag flows appear to be more general examples of the classical 

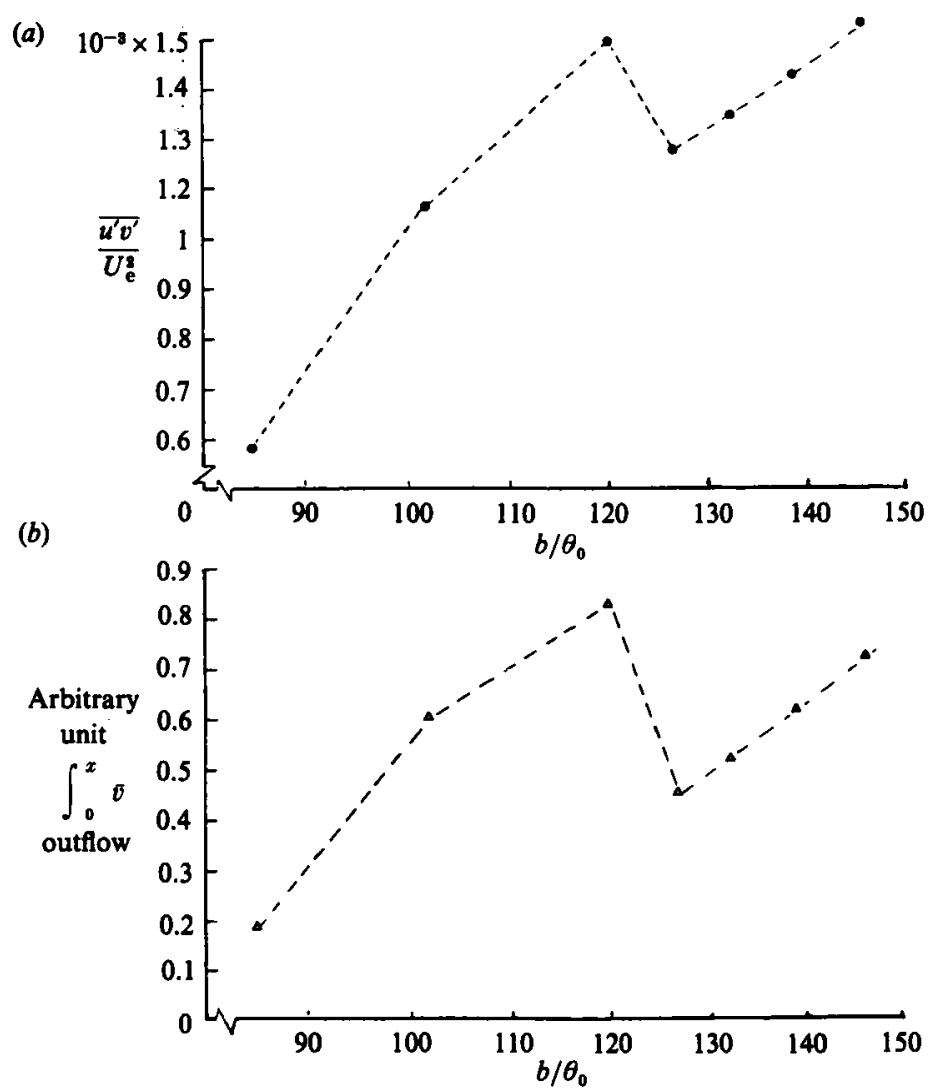

Figure 25. (a) Typical variation of the Reynolds stress $\left(\overline{u^{\prime} v^{\prime}} / U_{\mathrm{e}}^{2}\right)$ at a fixed $x / \theta_{0}$ with $b / \theta_{0}$. (b) Typical variation of the outflow from the cavity with $b / \theta_{0}$.

cavity flow depicted in figure 1. In fact, it is now clear that their 'low-drag' regime corresponds to the regime for self-sustained cavity flow oscillations while their 'medium-drag' regime corresponds to what we now call the cavity 'wake mode' (figure 24), and the corresponding terms will be used synonymously. The correspondence in the two experiments is remarkably good, considering the difference in the geometries and in Reynolds number $\left(8 \times 10^{5}\right.$ and $2 \times 10^{4}$, respectively).

The flow visualizations and the flow-field measurements presented in the preceding sections show that in the low-drag regime the cavity shear layer always stagnates at the downstream corner, even with increasing gap width. Only in the wake mode does it stagnate fully below the corner, increasingly so with increasing gap width. We believe that the tendency for the flow to stagnate at the downstream corner in the self-sustained modes is a result of a regulating mechanism, for which we propose the following tentative argument.

The amplitude of the oscillations as they amplify along the shear layer depends on the initial amplitude at the upstream corner, which in turn depends on the strength of the feedback from the downstream corner, where the shear layer impinges. This equilibrium is stable. If it is disturbed by a shift in the mean-stagnation-point location the feedback signal will be reduced. This seems obvious for displacement outward from the cavity but it is also true for inward displacement, as demonstrated by Rockwell \& Knisely (1979) who found that an inward displacement of $6 \theta_{0}$ reduced 
the transverse fluctuation amplitude by half. Now an inward shift of the stagnation point implies an increased inflow into the cavity at the downstream end, expressed by

$$
\int_{x^{*}}^{b} \bar{v}(x, 0) \mathrm{d} x,
$$

for negative values of $\bar{v}$; but this must be equalled by an increase in the outflow,

$$
\int_{0}^{x^{*}} \bar{v}(x, 0) \mathrm{d} x,
$$

over the upstream portion of the cavity mouth and implies an increase in the level of Reynolds stress (figure 25). This is not consistent with the reduced feedback signal and implies that the stagnation point will return to its 'lock-on' position because the required outflow-inflow level cannot be sustained. Actually, the level of the feedback signal is determined by the position of the shear-layer vortices as they impinge on the downstream corner as shown by Rockwell \& Knisely (1979); it seems this level is maximum when, in the mean flow, the dividing streamline reattaches on the corner.

The existence of coupling between outflow from the cavity and the deflection of the shear layer near the downstream corner was demonstrated by Sarohia \& Massier (1976) who found that external mass injection (bleed flow) into the cavity causes an outward deflection of the shear layer near the downstream corner, as expected, and a reduction in the level of fluctuation.

To summarize, the mechanism for stable equilibrium of the shear layer with 'lock-on' to the downstream corner may be explained as follows. An inward displacement of the shear layer at that corner results in a lowering of feedback signal and thus of Reynolds stress. This decreases the entrainment in the initial portion of the shear layer and reduces the positive outflow. Correspondingly the inflow at the downstream end must be reduced and the shear layer returns to its initial position.

Although this proposed self-regulating mechanism for the low-drag regime has not been demonstrated explicitly, our qualitative observations of the flow in the regime of self-sustained oscillation indicate that the flow is very stable and resistant to moderate external disturbances until the gap becomes so large that the wake-mode instability can take over. That instability can be delayed a little by controlled, external reinforcement of the self-sustained oscillations (Gharib 1983, 1987) and the range of the low-drag regime correspondingly increased.

In the wake mode there is large-scale motion across the centreline. Therefore this mode could be suppressed, in two-dimensional flow, by placing a splitter plate along the axis, but in our axially symmetric flow this possibility does not exist (the axisymmetric sting, topologically different from the two-dimensional splitter plate, does not cut off communication between the two sides of the flow).

The research described in this paper was carried out at the Jet Propulsion Laboratory, California Institute of Technology and was sponsored by the US Army Research Office and the National Aeronautics and Space Administration. Support was also received from the National Science Foundation. Valuable discussions of aspects of the research with V. Sarohia and D. Rockwell are also acknowledged. 


\section{REFERENCES}

Bradshaw, P. 1966 The effect of initial conditions on the development of a free shear layer. J. Fluid Mech. 26, 225.

Browand, F. K. 1966 An experimental investigation of the instability of an incompressible, separated shear layer. J. Fluid Mech. 26, 281.

Dewey, C. F. 1976 Qualitative and quantitative flow field visualization utilizing laser-induced fluorescence. $A G A R D-C P-193,17-1$.

Dimotakis, P. E., Miake-Lye, R. C. \& Papantoniou, D. A. 1983 Structure and dynamics of round turbulent jets. Phys. Fluids 26 (11), 3185.

Dimotakis, P. E. \& Brown, G. L. 1976 The mixing layer at high Reynolds number; largestructure dynamics and entrainment. J. Fluid Mech. 78 (3), 535.

Fox, J. 1968 A criterion for the transition between flow regimes in turbulent cavity flow. AIAA J. $4(2), 364$.

Freymuth, P. 1966 On transition in a separated laminar boundary layer. J. Fluid Mech. 25, 683.

GHARIB, M. 1983 The effect of flow oscillations on cavity drag and a technique for its control. Ph.D. thesis, California Institute of Technology, Pasadena, CA.

GHARIB, M. 1987 Response of the cavity shear layer oscillations to external forcing. AIAAJ. $25,43$.

Ho, C. M. \& Huana, L. S. 1982 Subharmonics and vortex merging in mixing layers. J. Fluid Mech. $119,443$.

Karamchet, K. 1956 Sound radiated from surface cutouts in high-speed flows. Ph.D. thesis, California Institute of Technology, Pasadena, CA.

KistLer, A. L. \& TAN, F. C. 1967 Some properties of turbulent separated flows. Phys. Fluids Suppl. 165.

KNISELY, C. W. 1980 An experimental investigation of low frequency self-modulation of incompressible impinging cavity shear layers. Ph.D. dissertation, Lehigh University, Bethlehem, PA.

Koenia, K. \& Roshko, A. 1985 An experimental study of geometrical effects on the drag and flow field of two bluff bodies separated by a gap. J. Fluid Mech. 156, 167.

LIEPMANN, H. W. \& LAUFER, J. 1947 Invetigations of free turbulent mixing. NACA Tech. Note no. 1257.

MichaLKe, A. 1965 On spatially growing disturbances in an inviscid shear layer. J. Fluid Mech. 23, 521 .

Oster, D. \& Wygnanski, I. 1982 The forced mixing layer between parallel streams. J. Fluid Mech. 123, 91.

Rockwell, D. \& KNisely, C. 1979 The organized nature of flow impingement upon a corner. J. Fluid Mech. 93, 413.

Rockwell, D. \& NaUdascher, E. 1978 Review self-sustaining oscillations of flow past cavities. Trans. ASME I: J. Fluid Engng 100, 152.

Roshko, A. 1955 Some measurements of flow in a rectangular cutout. NACA TN 3488.

Sarohis, V. 1975 Experimental and analytical investigation of oscillations in flows over cavities. $\mathrm{Ph}$.D. thesis, California Institute of Technology, Pasadena, CA.

Sarohia, V. \& Massier, P. E. 1976 Control of cavity noise. AIAA Paper 76-528. Presented at 3rd AIAA Aero-Acoustics Conf., Palo Alto, 20-23 July, 1976.

SARohia, V. \& MASSIER, P. E. 1977 Investigation of pressure oscillations in axi-symmetric cavity flows. Harry Diamond Laboratories Rpt. HDL-CR-77-025-1.

StUaRT, J. T. 1967 On finite amplitude oscillations in laminar mixing layers. J. Fluid Mech. 29, 417.

WERLE, H. 1974 Le tunnel hydrodynamique au service de la recherche aerospatiale. Publ.no. 156, ONERA, France. 
Woolley, J. P. \& Karamcheti, K. 1973 The role of jet stability in edgetone generation. AIAA Paper 73-628, AIAA 6th Fluid and Plasma Dynamics Conf. 16-18 July, Palm Springs, CA.

Wygnanski, I. \& Fiedler, H. E. 1970 The two-dimensional mixing region. J. Fluid Mech. 41, 327.

ZIADA, S. \& Rock WELL, D. 1981 Generation of higher harmonics in a self-oscillating mixing layer edge system. AIAA J. 20 (2), 196. 